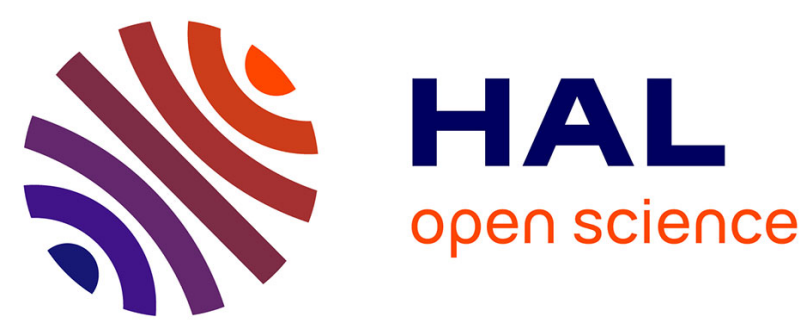

\title{
The Role of Nonconservative Interactions in the Asymptotic Limit of Thermostatted Kinetic Models
}

\author{
C. Bianca, C. Dogbe, A. Lemarchand
}

\section{To cite this version:}

C. Bianca, C. Dogbe, A. Lemarchand. The Role of Nonconservative Interactions in the Asymptotic Limit of Thermostatted Kinetic Models. Acta Applicandae Mathematicae, 2015, 139 (1), pp.1-24. 10.1007/s10440-014-9967-z . hal-01205183

\section{HAL Id: hal-01205183 \\ https://hal.sorbonne-universite.fr/hal-01205183}

Submitted on 25 Sep 2015

HAL is a multi-disciplinary open access archive for the deposit and dissemination of scientific research documents, whether they are published or not. The documents may come from teaching and research institutions in France or abroad, or from public or private research centers.
L'archive ouverte pluridisciplinaire HAL, est destinée au dépôt et à la diffusion de documents scientifiques de niveau recherche, publiés ou non, émanant des établissements d'enseignement et de recherche français ou étrangers, des laboratoires publics ou privés. 


\title{
The role of nonconservative interactions in the asymptotic limit of thermostatted kinetic models
}

\author{
Carlo Bianca $^{1,2}$, Christian Dogbe ${ }^{3}$ and Annie Lemarchand ${ }^{1,2}$ \\ ${ }^{1}$ Sorbonne Universités, UPMC Univ Paris 06, UMR 7600, \\ Laboratoire de Physique Théorique de la Matière Condensée, \\ 4, place Jussieu, case courrier 121, 75252 Paris Cedex 05, France \\ ${ }^{2}$ CNRS, UMR 7600 LPTMC, Paris, France \\ ${ }^{3}$ Université de Caen, LMNO, CNRS, UMR 6139, \\ Department of Mathematics, 14032 Caen Cedex, France
}

July 29, 2014

\begin{abstract}
This paper is concerned with the asymptotic analysis of space-velocity dependent thermostatted kinetic frameworks which include conservative, nonconservative and stochastic operators. The mathematical frameworks are integro-partial differential equations that can be proposed for the modeling of most phenomena occurring in biological and chemical systems. Specifically the paper focuses on the derivation of macroscopic equations obtained by performing a low-field and a high-field scaling into the thermostatted kinetic framework and considering the related convergence when the scaling parameter goes to zero. In the low-field limit, the macroscopic equations show diffusion with respect to both the space variable and a scalar variable that is introduced for the modeling of the strategy of the particle system. In the high-field limit, the macroscopic equations show hyperbolic behavior. The asymptotic analysis is also generalized to systems decomposed in various functional subsystems.
\end{abstract}

2000 Mathematics Subject Classification. 35Q20, 82C22, 92B05

Keywords and phrases. Dynamical Systems, Thermostats, Nonlinearity, Low-field and High-field scaling, Macroscopic equation

\section{Introduction}

The development of modern technologies and the related computational models have allowed a complete description of complex phenomena that appear in most biological and chemical systems at different scales: atomic, molecular, genetic, cellular, organ, tissue. Specifically, in most of the computational and mathematical models of the pertinent literature, the description has been performed at a specific scale without considering the problem to link the different phenomena occurring at different scales.

In particular, mathematical models have been employed for a complete description of various phenomena occurring in complex living systems. In detail, at atomic/molecular/genetic scales, mathematicians have proposed ODE-based models (see the review paper [1]); at the 
cellular/organ scale the generalized kinetic theory approach has been applied (see the book [2] and references cited therein, and also the paper [3]); at tissue scale the continuum mechanics approach has been considered (see the book [4] and references cited therein, and papers $[5,6])$. These models have been derived by considering only the phenomenological description occurring at a specific scale without taking care of the phenomena at a lower scale.

However, mathematicians have developed an analytical method, called asymptotic method, for deriving macroscopic equations fulfilled by the local macroscopic quantities (density and momenta of the distribution function) by asymptotic limits of kinetic models, see among others, the papers $[7,8,9,10,11,12,13,14,15,16,17,18,19,20,21]$.

The asymptotic method consists in defining the appropriate time and space scaling, which is a complex task considering that the ratio between the various rates characterizing each evolution plays an important role in assessing the structure of mathematical macroscopic equations derived from the kinetic equations. Therefore the macroscopic models are derived by letting the inter-particle distances tend to those of the macroscopic level. In particular, in the pertinent literature, there exists the so-called low-field and high-field limits, see the reference section of the book [2] and [22, 23]. In particular the low-field asymptotic limit of kinetic equations leads to a parabolic (reaction-diffusion) equation and the high-field limit leads to hyperbolic equations. This is the investigation that is pursued into the present paper.

The kinetic framework considered in this paper belongs to the class of thermostatted kinetic models that have been recently proposed in papers $[24,25,26]$ for the modeling of complex systems in physics and life sciences. Specifically the system is composed by a large number of particles whose microscopic state includes not only space and velocity variables but also a scalar variable, usually called activity, related to the function expressed by the particles that in this context are called active particles or agents. The time evolution of the system is described by a distribution function defined onto the macroscopic state of the particles. Mutual interactions among the particles modify the asymptotic evolution of the system. The mathematical framework, which belongs to the class of integro-partial differential equations, includes conservative, nonconservative and stochastic operators (in particular a velocity-jump process). Moreover a mathematical thermostat, in accordance with the Gauss principle of least constrain [27], is introduced for controlling the increase of the kinetic or activity energy of the system.

Specifically the present paper focuses on the derivation of macroscopic equations by performing a low-field and a high-field scaling and, under some technical assumptions on the conservative, nonconservative and the stochastic (velocity-jump) operators, the related convergence of the asymptotic limit, when the scaling parameter goes to zero, is reached. In the low-field limit, the macroscopic equations show diffusion with respect to both the space and activity variables. In the high-field limit, the macroscopic equations show hyperbolic behavior. The asymptotic analysis is also generalized to complex systems decomposed into a large number of functional subsystems.

It is worth precising that the asymptotic method is also applied to the $p$-thermostatted kinetic framework proposed in the paper [24], which is a new thermostatted mathematical framework where the $p$-thermostat has been introduced for the control of the time evolution of higher-order momenta.

The analysis developed in this paper is based on the method developed in papers [28, 29] (see also the reference section for a deeper understanding of the method) where the authors have considered a thermostatted kinetic framework with only conservative interactions and a velocity-jump process.

It is worth stressing that, to the best of our knowledge, this is the first paper that attempts to perform the asymptotic method, under the low-field scaling, to the thermostatted kinetic 
framework and to the $p$-thermostatted kinetic framework with nonconservative interactions. In particular the results show what are the effects of nonconservative interactions at the macroscopic scale and how these types of interactions modify the terms into the macroscopic equation. These results are of great interest in the modeling of the complex biological and chemical systems where proliferation and destruction of particles occur, such as in cancerimmune system competition, see [30, 31, 32, 33, 34].

The present paper is organized into five more sections which follow this introduction. In detail, Section 2 introduces the generalized thermostatted kinetic framework for the modeling of systems composed by particles whose microscopic state includes also the scalar variable related to the strategy of the particles (activity). The active particles are also able to proliferate and their velocity is described by a jump process. The mathematical frameworks are coupled with a thermostat and a $p$-thermostat. Preliminary properties of the operators which define the mathematical framework are presented in Section 2.1. Section 3 is meant to the definition of the low-field scaling, the scaled thermostatted kinetic frameworks, and the statement and proof of one of the main results of the present paper that consists of the derivation of the macroscopic equations for the local density of the system. A specific model is considered in Section 4; the macroscopic equation for the local density is derived for the relaxation model. Section 5 is concerned with the high-field scaling and the derivation of the macroscopic equations for the local density and momentum of the system. Finally Section 6 concludes the paper with some technical generalizations related to the complex biological and chemical systems characterized by a large number of active particles grouped into functional subsystems according to a recent system biology approach. Future research perspectives are also discussed within this section.

\section{Generalized thermostatted kinetic frameworks}

This section is concerned with the definition of the thermostatted kinetic equations that are the underlying frameworks for the derivation of the macroscopic equations obtained by the asymptotic limit method. The mathematical framework refers to the modeling of the complex biological/chemical system that is a set of interacting particles having a specific function whose magnitude is taken into account by introducing a scalar variable $u$ called activity. The magnitude of the activity variable is modified by the conservative interactions that are defined by considering the encounter rate among very close particles and the probability that particles change their activity. Birth and death processes are also considered by defining the proliferative and destructive rates. The microscopic state of the particles also includes space $\mathbf{x}$ and velocity $\mathbf{v}$ variables; in particular the space variable is not modified by the interactions and the velocity variable changes according to a jump process.

Let $f=f(t, \mathbf{x}, \mathbf{v}, u)$ be the distribution function of the particles of the system having at time $t$ the microscopic state $(\mathbf{x}, \mathbf{v}, u)$, where $(\mathbf{x}, \mathbf{v}) \in D_{\mathbf{x}} \times D_{\mathbf{v}} \subset \mathbb{R}^{3} \times \mathbb{R}^{3}$ and $u \in D_{u} \subset \mathbb{R}$. The system is subjected to a known external force field $F=F(u): D_{u} \rightarrow \mathbb{R}$ that moves the system out of equilibrium.

The derivation of the thermostatted framework requires the definition of the following main quantities:

(i) The interaction rate $\eta\left(u_{*}, u^{*}\right)$, which denotes the probability that a particle with microscopic state $\left(\mathbf{x}, \mathbf{v}, u_{*}\right)$ interacts with a particle with microscopic state $\left(\mathbf{x}, \mathbf{v}, u^{*}\right)$.

(ii) The particles with microscopic state $\left(\mathbf{x}, \mathbf{v}, u_{*}\right)$ interacting with the particles with microscopic state $\left(\mathbf{x}, \mathbf{v}, u^{*}\right)$ have a probability density to reach the microscopic state $(\mathbf{x}, \mathbf{v}, u)$ given by $\mathscr{A}=\mathscr{A}\left(u_{*}, u^{*}, u\right): D_{u} \times D_{u} \times D_{u} \rightarrow \mathbb{R}^{+}$such that $\|\mathscr{A}\|_{L^{1}\left(D_{u}\right)}=1, \forall u_{*}, u^{*} \in$ $D_{u}$. 
(iii) The velocity variable $\mathbf{v}$ that belongs to the domain $D_{\mathbf{v}}$ assumed bounded and spherically symmetric with respect to $(0,0,0)$, changes according to a stochastic velocity-jump process in which the particle moves with constant speed describing a straight line whose direction changes continuously.

The evolution equation for the distribution function $f$ is obtained by equating the time derivative of $f$ to the balance of the inlet and outlet flows into the elementary volume of the space of the microscopic states. Specifically, denoting by $\partial_{t}, \nabla_{\mathbf{x}}$ the time derivative and the space gradient of $f$, respectively, and by $\cdot$, the canonic scalar product in $\mathbb{R}^{3}$ between two vectors, the thermostatted kinetic framework for $f$ thus reads:

$$
\left(\partial_{t}+\mathbf{v} \cdot \nabla_{\mathbf{x}}\right) f+\partial_{u}\left(F(u)\left(1-u \int_{\Omega} u f d \mathbf{x} d \mathbf{v} d u\right) f\right)=J[f]+N[f]+\nu V[f]
$$

where $\Omega=D_{\mathbf{x}} \times D_{\mathbf{v}} \times D_{u}$ and

- $\mathbf{v} \cdot \nabla_{\mathbf{x}} f$ is the transport operator;

- $\nu$ is the turning rate or turning frequency of the velocity-jump, hence $1 / \nu$ is the mean run time;

- The conservative operator $J[f]=J[f](t, \mathbf{x}, \mathbf{v}, u)=G[f](t, \mathbf{x}, \mathbf{v}, u)+L[f](t, \mathbf{x}, \mathbf{v}, u)$, which models the conservative changing of the activity, reads:

$$
\begin{aligned}
G[f] & =\int_{D_{u} \times D_{u}} \eta\left(u_{*}, u^{*}\right) \mathscr{A}\left(u_{*}, u^{*}, u\right) f\left(t, \mathbf{x}, \mathbf{v}, u_{*}\right) f\left(t, \mathbf{x}, \mathbf{v}, u^{*}\right) d u_{*} d u^{*} \\
L[f] & =-f(t, \mathbf{x}, \mathbf{v}, u) \int_{D_{u}} \eta\left(u_{*}, u^{*}\right) f\left(t, \mathbf{x}, \mathbf{v}, u^{*}\right) d u^{*}
\end{aligned}
$$

that models the gain of particles with microscopic state $(\mathbf{x}, \mathbf{v}, u)$, due to activity transitions of the particles with microscopic state $\left(\mathbf{x}, \mathbf{v}, u_{*}\right)$ that interact with particles having the microscopic state $\left(\mathbf{x}, \mathbf{v}, u^{*}\right)$, and the loss of particles due to transitions into different microscopic states.

- The nonconservative operator $N[f]=N[f](t, \mathbf{x}, \mathbf{v}, u)$, which models proliferation or destruction of particles with microscoscopic state $(\mathbf{x}, \mathbf{v}, u)$, reads:

$$
N[f]=f(t, \mathbf{x}, \mathbf{v}, u) \int_{D_{u}} \eta\left(u_{*}, u^{*}\right) \mu\left(u_{*}, u^{*}\right) f\left(t, \mathbf{x}, \mathbf{v}, u^{*}\right) d u^{*} .
$$

where $\mu_{i j}$ denotes the net proliferative/destructive rate.

- The turning operator $V[f] \equiv V[f](t, \mathbf{x}, \mathbf{v}, u)$, which models the velocity-jump process, is the stochastic term defined as follows:

$$
V[f]=\int_{D_{\mathbf{v}}}\left[T\left(\mathbf{v}^{*}, \mathbf{v}\right) f\left(t, \mathbf{x}, \mathbf{v}^{*}, u\right)-T\left(\mathbf{v}, \mathbf{v}^{*}\right) f(t, \mathbf{x}, \mathbf{v}, u)\right] d \mathbf{v}^{*},
$$

where $T\left(\mathbf{v}^{*}, \mathbf{v}\right)$ is the turning kernel which gives the probability that the velocity $\mathbf{v}^{*} \in D_{\mathbf{v}}$ jumps into the velocity $\mathbf{v} \in D_{\mathbf{v}}$ (if a jump occurs).

- The thermostatted term reads:

$$
\mathscr{T}_{F}[f]:=\partial_{u}\left(F(u)\left(1-u \int_{\Omega} u f(t, \mathbf{x}, \mathbf{v}, u) d \mathbf{x} d \mathbf{v} d u\right) f(t, \mathbf{x}, \mathbf{v}, u)\right)
$$

which is a transport term due to the activity of the particles; the reader interested in Gaussian thermostats is referred to papers [27, 35, 36]. 
The turning event is usually governed by a Poisson process [12, 37]. The choice of a new velocity is independent of the run time. In general $\nu$ may be space-dependent and may depend on internal and external variables as well. Similar stochastic rotations of the velocity of the particles have been also introduced to simulate multiple collision dynamics in order to account for the effect of the solvent, see [38].

The role of the thermostat into the framework (2.1) is that of controling the second moment of local activity which is defined as follows:

$$
\mathbb{E}_{2}[f](t, \mathbf{x}, \mathbf{v})=\int_{D_{u}} u^{2} f(t, \mathbf{x}, \mathbf{v}, u) d u
$$

High-order local quantities are calculated if motivated by some interest for the modelling, see [18]. Indeed a generalization of the thermostatted framework (2.1) has been considered in paper [24] for controling the following $p$ th-order moment of local activity:

$$
\mathbb{E}_{p}[f](t, \mathbf{x}, \mathbf{v})=\int_{D_{u}} u^{p} f(t, \mathbf{x}, \mathbf{v}, u) d u, \quad p \in \mathbb{N} .
$$

This generalization, called the p-thermostatted kinetic framework, reads:

$$
\left(\partial_{t}+\mathbf{v} \cdot \nabla_{\mathbf{x}}\right) f+\partial_{u}\left(F(u)\left(1-u \int_{\Omega} u^{p-1} f d \mathbf{x} d \mathbf{v} d u\right) f\right)=J[f]+N[f]+\nu V[f]
$$

where the meaning of each term into the above equation can be recovered by the previous considerations. In this case the thermostat term reads:

$$
\mathscr{T}_{F, p}[f]:=\partial_{u}\left(F(u)\left(1-u \int_{\Omega} u^{p-1} f(t, \mathbf{x}, \mathbf{v}, u) d \mathbf{x} d \mathbf{v} d u\right) f(t, \mathbf{x}, \mathbf{v}, u)\right) .
$$

In the low field limit, the analysis is addressed to obtain the evolution equation of the local density $\varrho[f](t, \mathbf{x}, u)$ of the system defined at time $t$ in the position $\mathbf{x}$ and activity $u$, as follows:

$$
\varrho:=\varrho[f](t, \mathbf{x}, u)=\int_{D_{\mathbf{v}}} f(t, \mathbf{x}, \mathbf{v}, u) d \mathbf{v} .
$$

In the high-field limit, the main aim of this paper is to obtain the evolution equation of $\varrho[f]$ and of the relative mass velocity of particles $\mathbb{U}(t, \mathbf{x}, u)$ defined on $\left[0, \infty\left[\times D_{\mathbf{x}} \times D_{u}\right.\right.$ by

$$
\mathbb{U}:=\mathbb{U}[f](t, \mathbf{x}, u)=\frac{1}{\varrho[f](t, \mathbf{x}, u)} \int_{D_{\mathbf{v}}} \mathbf{v} f(t, \mathbf{x}, \mathbf{v}, u) d \mathbf{v} .
$$

The following pressure term

$$
\mathbb{P}:=\mathbb{P}[f](t, \mathbf{x}, u)=\int_{D_{\mathbf{v}}}(\mathbf{v}-\mathbb{U}) \otimes(\mathbf{v}-\mathbb{U}) f(t, \mathbf{x}, \mathbf{v}, u) d \mathbf{v},
$$

will be also taken into account.

\subsection{Preliminary properties of the operators}

This section deals with some preliminary properties of the consevative, nonconservative and velocity-jump operators. 
Lemma 2.1. If the interaction rate $\eta$ is assumed constant and the probability density $\mathscr{A}$ satisfies, for all $u_{*}, u^{*} \in D_{u}$, the following assumptions:

$$
\begin{gathered}
\int_{D_{u}} \mathscr{A}\left(u_{*}, u^{*}, u\right) d u=1, \\
\int_{D_{u}} u \mathscr{A}\left(u_{*}, u^{*}, u\right) d u=0, \\
\int_{D_{u}} u^{2} \mathscr{A}\left(u_{*}, u^{*}, u\right) d u=u_{*}^{2},
\end{gathered}
$$

then the gain operator

$$
G[f]=\eta \int_{D_{u} \times D_{u}} \mathscr{A}\left(u_{*}, u^{*}, u\right) f\left(t, \mathbf{x}, \mathbf{v}, u_{*}\right) f\left(t, \mathbf{x}, \mathbf{v}, u^{*}\right) d u_{*} d u^{*}
$$

satisfies, for all functions $f$, the following identities:

$$
\begin{gathered}
\int_{D_{u}} G[f](t, \mathbf{x}, \mathbf{v}, u) d u=\eta\left(\int_{D_{u}} f(t, \mathbf{x}, \mathbf{v}, u) d u\right)^{2}, \\
\int_{D_{u}} u G[f](t, \mathbf{x}, \mathbf{v}, u) d u=0, \\
\int_{D_{u}} u^{2} G[f](t, \mathbf{x}, \mathbf{v}, u) d u=\eta\left(\int_{D_{u}} f(t, \mathbf{x}, \mathbf{v}, u) d u\right)\left(\int_{D_{u}} u^{2} f(t, \mathbf{x}, \mathbf{v}, u) d u\right) .
\end{gathered}
$$

Proof. The condition (2.14) implies:

$$
\begin{aligned}
\int_{D_{u}} G[f](t, \mathbf{x}, \mathbf{v}, u) d u & =\int_{D_{u}}\left(\int_{D_{u} \times D_{u}} \eta \mathscr{A}\left(u_{*}, u^{*}, u\right) f\left(t, \mathbf{x}, \mathbf{v}, u_{*}\right) f\left(t, \mathbf{x}, \mathbf{v}, u^{*}\right) d u_{*} d u^{*}\right) d u \\
& =\int_{D_{u} \times D_{u}} \eta\left(\int_{D_{u}} \mathscr{A}\left(u_{*}, u^{*}, u\right) d u\right) f\left(t, \mathbf{x}, \mathbf{v}, u_{*}\right) f\left(t, \mathbf{x}, \mathbf{v}, u^{*}\right) d u_{*} d u^{*} \\
& =\eta\left(\int_{D_{u}} f\left(t, \mathbf{x}, \mathbf{v}, u_{*}\right) d u^{*}\right)\left(\int_{D_{u}} f\left(t, \mathbf{x}, \mathbf{v}, u^{*}\right) d u^{*}\right)
\end{aligned}
$$

Bearing in mind the condition (2.15) we have:

$$
\begin{aligned}
\int_{D_{u}} u G[f](t, u) d u & =\int_{D_{u}} u\left(\int_{D_{u} \times D_{u}} \eta \mathscr{A}\left(u_{*}, u^{*}, u\right) f\left(t, \mathbf{x}, \mathbf{v}, u_{*}\right) f\left(t, \mathbf{x}, \mathbf{v}, u^{*}\right) d u_{*} d u^{*}\right) d u \\
& =\int_{D_{u} \times D_{u}} \eta\left(\int_{D_{u}} u \mathscr{A}\left(u_{*}, u^{*}, u\right) d u\right) f\left(t, \mathbf{x}, \mathbf{v}, u_{*}\right) f\left(t, \mathbf{x}, \mathbf{v}, u^{*}\right) d u_{*} d u^{*} \\
& =0 .
\end{aligned}
$$

Finally the condition (2.16) implies that the following identity holds true:

$$
\begin{aligned}
\int_{D_{u}} u^{2} G[f](t, u) d u & =\int_{D_{u}} u^{2}\left(\int_{D_{u} \times D_{u}} \eta \mathscr{A}\left(u_{*}, u^{*}, u\right) f\left(t, \mathbf{x}, \mathbf{v}, u_{*}\right) f\left(t, \mathbf{x}, \mathbf{v}, u^{*}\right) d u_{*} d u^{*}\right) d u \\
& =\int_{D_{u} \times D_{u}} \eta\left(\int_{D_{u}} u^{2} \mathscr{A}\left(u_{*}, u^{*}, u\right) d u\right) f\left(t, \mathbf{x}, \mathbf{v}, u_{*}\right) f\left(t, \mathbf{x}, \mathbf{v}, u^{*}\right) d u_{*} d u^{*} \\
& =\eta \int_{D_{u} \times D_{u}} u_{*}^{2} f\left(t, \mathbf{x}, \mathbf{v}, u_{*}\right) f\left(t, \mathbf{x}, \mathbf{v}, u^{*}\right) d u_{*} d u^{*} \\
& =\eta\left(\int_{D_{u}} f\left(t, \mathbf{x}, \mathbf{v}, u^{*}\right) d u^{*}\right)\left(\int_{D_{u}} u_{*}^{2} f\left(t, \mathbf{x}, \mathbf{v}, u_{*}\right) d u_{*}\right)
\end{aligned}
$$


Therefore the proof of the Lemma is concluded.

The results in Lemma 2.1 carry out an important role in the existence of solution results for the relative Cauchy problem of the thermostatted kinetic frameworks (2.1) and (2.9). These results will not be reported here because they require further assumptions on the operators (see paper [25] for the existence result of solutions of the Cauchy problem for the thermostatted kinetic framework without nonconservative interactions). However in the case of the thermostatted kinetic frameworks (2.1) and (2.9), the global existence of solutions is not ensured in general. Moreover the validity of the Lemma 2.1 allows us to derive the evolution equation for the following first activity moment

$$
\int_{\Omega} u f(t, \mathbf{x}, \mathbf{v}, u) d \mathbf{x} d \mathbf{v} d u,
$$

even when nonconservative interactions are considered.

Following the proof of Lemma 2.1 is simple to obtain the proof of the following Lemma.

Lemma 2.2. If the interaction rate $\eta$ and the proliferative/destructive rate $\mu$ are assumed constants, then the loss operator and the nonconservative operators, respectively, satisfy the following identities:

$$
\begin{aligned}
& \int_{D_{u}} L[f](t, \mathbf{x}, \mathbf{v}, u) d u=-\eta\left(\int_{D_{u}} f(t, \mathbf{x}, \mathbf{v}, u) d u\right)^{2}, \\
& \int_{D_{u}} N[f](t, \mathbf{x}, \mathbf{v}, u) d u=\eta \mu\left(\int_{D_{u}} f(t, \mathbf{x}, \mathbf{v}, u) d u\right)^{2} .
\end{aligned}
$$

The asymptotic analysis, that will be performed in the present paper, strongly depends on the turning operator $V[f]$. Therefore the following Assumptions and Lemma are essential for the asymptotic limit of the thermostat frameworks (2.1) and (2.9).

The main properties of $V[f]$ are summarized below:

Lemma 2.3. Assume that the turning operator $V[f]$ given by Eq. (2.5) satisfies the following assumptions:

$\left(\boldsymbol{A}_{1}\right)$ Conservation of particles:

$$
\int_{D_{\mathbf{v}}} V[f] d \mathbf{v}=\int_{D_{\mathbf{v}}} \mathbf{v} V[f] d \mathbf{v}=0 .
$$

$\left(\boldsymbol{A}_{2}\right)$ There exists a bounded equilibrium velocity distribution $G(\mathbf{v}): D_{\mathbf{v}} \rightarrow \mathbb{R}^{+}$, independent of $t$ and $\mathbf{x}$, such that:

$$
T\left(\mathbf{v}^{*}, \mathbf{v}\right) G(\mathbf{v})=T\left(\mathbf{v}, \mathbf{v}^{*}\right) G\left(\mathbf{v}^{*}\right) .
$$

and

$$
\int_{D_{\mathbf{v}}} \mathbf{v} G(\mathbf{v}) d \mathbf{v}=0, \quad \int_{D_{\mathbf{v}}} G(\mathbf{v}) d \mathbf{v}=1 .
$$

$\left(\boldsymbol{A}_{3}\right)$ The kernel $T\left(\mathbf{v}, \mathbf{v}^{*}\right)$ is bounded, and there exists a constant $\sigma>0$ such that

$$
T\left(\mathbf{v}, \mathbf{v}^{*}\right) \geq \sigma G(\mathbf{v}), \quad \forall\left(\mathbf{v}, \mathbf{v}^{*}\right) \in D_{\mathbf{v}} \times D_{\mathbf{v}} .
$$

Then 
(i) For $f \in L^{2}\left(D_{\mathbf{v}} ; d \mathbf{v} / G\right)$, the integral equation $V[g]=f$ has a unique solution $g \in L^{2}\left(D_{\mathbf{v}} ; d \mathbf{v} / G\right)$ such that

$$
\int_{D_{\mathbf{v}}} g(\mathbf{v}) d \mathbf{v}=0 \text { if and only if } \int_{D_{\mathbf{v}}} f(\mathbf{v}) d \mathbf{v}=0 .
$$

(ii) The integral operator $V$ defines a self-adjoint Fredholm operator on the space $L^{2}\left(D_{\mathbf{v}} ; d \mathbf{v} / G\right)$.

(iii) The equation $V[f]=\mathbf{v} G(\mathbf{v})$ has a unique solution given by $f(\mathbf{v})=\chi(\mathbf{v})$.

(iv) The null-space of $V$ is spanned by a unique normalized and nonnegative function $G(\mathbf{v})$ :

$$
\operatorname{Ker}(V)=\operatorname{Span}\{G\} .
$$

The reader interested into the proof of the Lemma 2.3 is referred to paper [37].

It is worth stressing that the assumption $\left(\mathbf{A}_{2}\right)$ means that the flow produced by the equilibrium distribution $G(\mathbf{v})$ vanishes, and that $G$ is normalized with respect to the velocity domain. The function $\chi(\mathbf{v})$ gives the coefficient in the low-field limit equation.

\section{The macroscopic framework by low-field scaling}

This section aims at performing the asymptotic limit of the thermostatted kinetic frameworks (2.1) and (2.9) by a low-field scaling (low-field asymptotic limit). Specifically we consider the scaled thermostatted kinetic frameworks whose solutions are assumed to be bounded in a space of functions where all needed convergence results will be true.

The Hilbert space $L^{2}\left(D_{\mathbf{v}}, d \mathbf{v}\right)$ endowed with the usual scalar product

$$
\langle f, g\rangle=\int_{D_{\mathbf{v}}} f(\mathbf{v}) g(\mathbf{v}) d \mathbf{v}, \quad f, g \in L^{2}\left(D_{\mathbf{v}}, d \mathbf{v}\right),
$$

will be used in the sequel and the average of the function $\varphi$ with respect to variable $\mathbf{v}$ will be denoted by

$$
\langle\varphi\rangle:=\langle\varphi, 1\rangle=\int_{D_{\mathbf{v}}} \varphi(\mathbf{v}) d \mathbf{v} .
$$

Moreover the following Kronecher delta will be used:

$$
\delta_{i j}=\left\{\begin{array}{cc}
1 & \text { if } i=j \\
0 & \text { if } i \neq j
\end{array}\right.
$$

To prove the main result an integral approach will be more suitable than Chapman-Enskog method. Indeed, employing test functions directly on conservation equations for the velocity moments of distribution function and balancing with care the various terms, we can pass to the limit of vanishing mean free path and hope to derive the macroscopic description.

\subsection{Low-field scaling and scaled thermostatted frameworks}

Let $\alpha$ be the microscopic length scale (mean free path), $\beta$ the macroscopic length scale, and $\varepsilon$ the ratio between $\alpha$ and $\beta$. In order to obtain the macroscopic equation from the kinetic frameworks, the following scaling is considered:

$$
(t, \mathbf{x}, \mathbf{v}, u, F) \rightarrow\left(\frac{t}{\varepsilon}, \mathbf{x}, \mathbf{v}, u, \varepsilon^{\ell} F\right), \quad \ell \geq 1,
$$


and considering the relationship that exists among the interaction rate, the proliferative/destructive rate and the turning frequency, the following choice of the rates can be set:

$$
\eta=\varepsilon^{r}, \quad \mu=\varepsilon^{q}, \quad \nu=\frac{1}{\varepsilon^{s}}
$$

where $q, r, s \geq 1$. Therefore the distribution function reads:

$$
f_{\varepsilon}(t, \mathbf{x}, \mathbf{v}, u)=f\left(\frac{t}{\varepsilon}, \mathbf{x}, \mathbf{v}, u\right) .
$$

Consequently the thermostatted framework (2.1) is scaled as follows:

$$
\varepsilon \partial_{t} f_{\varepsilon}+\mathbf{v} \cdot \nabla_{\mathbf{x}} f_{\varepsilon}+\varepsilon^{\ell} \mathscr{T}_{F}\left[f_{\varepsilon}\right]=\varepsilon^{r} \tilde{J}\left[f_{\varepsilon}\right]+\varepsilon^{r+q} \tilde{N}\left[f_{\varepsilon}\right]+\frac{1}{\varepsilon^{s}} \tilde{V}\left[f_{\varepsilon}\right]
$$

and the scaled $p$-thermostatted framework (2.9) reads:

$$
\varepsilon \partial_{t} f_{\varepsilon}+\mathbf{v} \cdot \nabla_{\mathbf{x}} f_{\varepsilon}+\varepsilon^{\ell} \mathscr{T}_{F, p}\left[f_{\varepsilon}\right]=\varepsilon^{r} \tilde{J}\left[f_{\varepsilon}\right]+\varepsilon^{r+q} \tilde{N}\left[f_{\varepsilon}\right]+\frac{1}{\varepsilon^{s}} \tilde{V}\left[f_{\varepsilon}\right]
$$

where $\tilde{J}\left[f_{\varepsilon}\right], \tilde{N}\left[f_{\varepsilon}\right], \tilde{V}\left[f_{\varepsilon}\right], \mathscr{T}_{F}\left[f_{\varepsilon}\right]$, and $\mathscr{T}_{F, p}\left[f_{\varepsilon}\right]$ are the scaled conservative, nonconservative, turning and thermostat operators, respectively, that read:

$$
\begin{aligned}
\tilde{J}\left[f_{\varepsilon}\right](t, \mathbf{x}, \mathbf{v}, u)= & \int_{D_{u} \times D_{u}} \mathscr{A}\left(u_{*}, u^{*}, u\right) f_{\varepsilon}\left(t, \mathbf{x}, \mathbf{v}, u_{*}\right) f_{\varepsilon}\left(t, \mathbf{x}, \mathbf{v}, u^{*}\right) d u_{*} d u^{*} \\
& -f_{\varepsilon}(t, \mathbf{x}, \mathbf{v}, u) \int_{D_{u}} f_{\varepsilon}\left(t, \mathbf{x}, \mathbf{v}, u^{*}\right) d u^{*} \\
\tilde{N}\left[f_{\varepsilon}\right]= & f_{\varepsilon}(t, \mathbf{x}, \mathbf{v}, u) \int_{D_{u}} f_{\varepsilon}\left(t, \mathbf{x}, \mathbf{v}, u^{*}\right) d u^{*}, \\
\tilde{V}\left[f_{\varepsilon}\right](t, \mathbf{x}, \mathbf{v}, u)= & \int_{D_{\mathbf{v}}}\left[T\left(\mathbf{v}^{*}, \mathbf{v}\right) f_{\varepsilon}\left(t, \mathbf{x}, \mathbf{v}^{*}, u\right)-T\left(\mathbf{v}, \mathbf{v}^{*}\right) f_{\varepsilon}(t, \mathbf{x}, \mathbf{v}, u)\right] d \mathbf{v}^{*} . \\
\mathscr{T}_{F}\left[f_{\varepsilon}\right]= & \partial_{u}\left(F(u)\left(1-u \int_{\Omega} u f_{\varepsilon}(t, \mathbf{x}, \mathbf{v}, u) d \mathbf{x} d \mathbf{v} d u\right) f_{\varepsilon}(t, \mathbf{x}, \mathbf{v}, u)\right) \\
\mathscr{T}_{F, p}\left[f_{\varepsilon}\right]= & \partial_{u}\left(F(u)\left(1-u \int_{\Omega} u^{p-1} f_{\varepsilon}(t, \mathbf{x}, \mathbf{v}, u) d \mathbf{x} d \mathbf{v} d u\right) f_{\varepsilon}(t, \mathbf{x}, \mathbf{v}, u)\right)
\end{aligned}
$$

The singular character of the problem is related to the fact that the small parameter $\varepsilon$ basically multiplies all derivatives in the equation (3.3). The following Lemma holds true.

Lemma 3.1. (Factorization of the function asymptotic limit) Let $f_{\varepsilon}(t, \mathbf{x}, \mathbf{v}, u)$ be a sequence of solutions of the scaled thermostatted kinetic equation (3.3) or (3.4). Assume that the turning operator $V$ satisfies the assumptions $\left(\boldsymbol{A}_{1}-\boldsymbol{A}_{2}-\boldsymbol{A}_{3}\right)$ and, when $\varepsilon \rightarrow 0$, the following statements hold true:

$$
\begin{aligned}
f_{\varepsilon} & \longrightarrow f \quad \text { a.e. in }[0, \infty) \times D_{\mathbf{x}} \times D_{\mathbf{v}} \times D_{u}, \\
\tilde{V}\left[f_{\varepsilon}\right] & \longrightarrow \tilde{V}[f]
\end{aligned}
$$

Then the asymptotic limit $f$ of the sequence $f_{\varepsilon}$ (modulo the extraction of a subsequence) admits the following factorization:

$$
f(t, \mathbf{x}, \mathbf{v}, u)=\varrho(t, \mathbf{x}, u) G(\mathbf{v}),
$$

where $\varrho$ is the local macroscopic density (2.11) of the system. 
Proof. To prove the Lemma, firstly we multiply Eq. (3.3) or Eq. (3.4) by $\varepsilon^{s}$ and we let $\varepsilon$ go to zero. Since, for $T>0, f_{\varepsilon}(t, \mathbf{x}, \mathbf{v}, u)$ is uniformly bounded in

$$
L^{\infty}\left[0, T ; L^{2}\left(D_{\mathbf{x}} \times D_{\mathbf{v}} \times D_{u}\right)\right]
$$

it weakly converges (modulo the extraction of a subsequence) to a solution $f_{\varepsilon}(t, \mathbf{x}, \mathbf{v}, u)$ of the equation

$$
\tilde{V}[f]=0 .
$$

Therefore we deduce from Lemma 2.3 the existence of a function $\varrho=\varrho(t, \mathbf{x}, u):\left[0, \infty\left[\times D_{\mathbf{x}} \times\right.\right.$ $D_{u} \rightarrow \mathbb{R}^{+}$independent of $\mathbf{v}$ and such that the relation (3.12) holds true. In particular

$$
\int_{D_{\mathbf{v}}} f(t, \mathbf{x}, \mathbf{v}, u) d \mathbf{v}=\int_{D_{\mathbf{v}}} \varrho(t, \mathbf{x}, u) G(\mathbf{v}) d \mathbf{v}=\varrho(t, \mathbf{x}, u) \int_{D_{\mathbf{v}}} G(\mathbf{v}) d \mathbf{v} .
$$

In order to calculate the asymptotic limit of the scaled thermostatted framework (3.3) or (3.4), we need to perform the limit, when $\varepsilon$ goes to zero, of the term

$$
\frac{1}{\varepsilon}\left\langle\mathbf{v} \cdot \nabla_{\mathbf{x}} f_{\varepsilon}\right\rangle
$$

Lemma 3.2. (The limit of the convective term). Let $f_{\varepsilon}(t, \mathbf{x}, \mathbf{v}, u)$ be a sequence of solutions of the scaled thermostatted kinetic equation (3.3) or (3.4). Assume that the turning operator $V$ satisfies the assumptions $\left(\boldsymbol{A}_{1}-\boldsymbol{A}_{2}-\boldsymbol{A}_{3}\right)$ and, when $\varepsilon \rightarrow 0$, the following statements hold true:

$$
\begin{aligned}
f_{\varepsilon} & \longrightarrow f \quad \text { a.e. in }[0, \infty) \times D_{\mathbf{x}} \times D_{\mathbf{v}} \times D_{u}, \\
\tilde{V}\left[f_{\varepsilon}\right] & \longrightarrow \tilde{V}[f],
\end{aligned}
$$

then

$$
\frac{1}{\varepsilon}\left\langle\mathbf{v} \cdot \nabla_{\mathbf{x}} f_{\varepsilon}\right\rangle \underset{\varepsilon \rightarrow 0}{\longrightarrow} \delta_{s, 1} d i v_{\mathbf{x}}\left\langle(\chi(\mathbf{v}) \otimes \mathbf{v}) \nabla_{\mathbf{x}} \varrho\right\rangle .
$$

where $\chi(\mathbf{v})$ is the only solution of the equation $\tilde{V}[f]=\mathbf{v} G(\mathbf{v})$.

Proof. According to Lemma 2.3, the term (3.15) can be rewritten as follows:

$$
\begin{aligned}
\frac{1}{\varepsilon}\left\langle\mathbf{v} \cdot \nabla_{\mathbf{x}} f_{\varepsilon}\right\rangle & =\operatorname{div}_{\mathbf{x}}\left\langle\frac{\mathbf{v} f_{\varepsilon}}{\varepsilon}\right\rangle \\
& =\frac{1}{\varepsilon} \operatorname{div}_{\mathbf{x}}\left\langle\mathbf{v} f_{\varepsilon} \frac{G(\mathbf{v})}{G(\mathbf{v})}\right\rangle=\operatorname{div}_{\mathbf{x}}\left\langle\frac{\tilde{V}\left[f_{\varepsilon}\right]}{\varepsilon} \frac{\chi(\mathbf{v})}{G(\mathbf{v})}\right\rangle .
\end{aligned}
$$

Moreover, multiplying the right-and-left-hand sides of Eq. (3.3) by $\varepsilon^{s-1}$, we obtain

$$
\frac{1}{\varepsilon} \tilde{V}\left[f_{\varepsilon}\right]=\varepsilon^{s} \partial_{t} f_{\varepsilon}+\varepsilon^{s+\ell-1} \mathscr{T}_{\mathscr{F}}\left[f_{\varepsilon}\right]+\varepsilon^{s-1} \mathbf{v} \cdot \nabla_{\mathbf{x}} f_{\varepsilon}-\varepsilon^{s+r-1} J\left[f_{\varepsilon}\right]-\varepsilon^{s+q+r-1} N\left[f_{\varepsilon}\right]:=\mathbf{L}\left[f_{\varepsilon}\right] .
$$

Therefore, plugging the above term into Eq. (3.19) gives

$$
\frac{1}{\varepsilon}\left\langle\mathbf{v} \cdot \nabla_{\mathbf{x}} f_{\varepsilon}\right\rangle=\operatorname{div}_{\mathbf{x}}\left\langle\mathbf{L}\left[f_{\varepsilon}\right] \frac{\chi(\mathbf{v})}{G(\mathbf{v})}\right\rangle .
$$


The assumption of convergence of $f_{\varepsilon}$ ensures the convergence in the sense of distributions of its moments, so that one can pass to the limit in the equation. Note that if $s=1$ then, when $\varepsilon \rightarrow 0$, one obtains

$$
\varepsilon^{s} \partial_{t} f_{\varepsilon}+\varepsilon^{s+\ell-1} \mathscr{T}_{\mathscr{F}}\left[f_{\varepsilon}\right]-\varepsilon^{s+r-1} \tilde{J}\left[f_{\varepsilon}\right]-\varepsilon^{s+q+r-1} \tilde{N}\left[f_{\varepsilon}\right] \rightarrow 0 \quad \text { and } \quad \varepsilon^{s-1} \mathbf{v} \cdot \nabla_{\mathbf{x}} f_{\varepsilon} \rightarrow \mathbf{v} \cdot \nabla_{\mathbf{x}} f .
$$

If $s>1$ then $\mathbf{L}\left[f_{\varepsilon}\right] \rightarrow 0$, when $\varepsilon \rightarrow 0$.

Therefore, if $s=1$, we have the following limit:

$$
\frac{1}{\varepsilon}\left\langle\mathbf{v} \cdot \nabla_{\mathbf{x}} f_{\varepsilon}\right\rangle \underset{\varepsilon \rightarrow 0}{\longrightarrow} \operatorname{div}_{\mathbf{x}}\left\langle\left(\frac{\chi(\mathbf{v})}{G(\mathbf{v})} \otimes \mathbf{v}\right) \nabla_{\mathbf{x}} f\right\rangle=\operatorname{div}_{\mathbf{x}}\left\langle(\chi(\mathbf{v}) \otimes \mathbf{v}) \nabla_{\mathbf{x}} \varrho\right\rangle .
$$

\subsection{The macroscopic equation}

Bearing all the previous sections in mind, the macroscopic equation which describes the evolution of the local macroscopic density $\varrho$ is stated in the following theorem.

Theorem 3.1. Let $f_{\varepsilon}(t, \mathbf{x}, \mathbf{v}, u)$ be a sequence of solutions of the scaled thermostatted kinetic equation (3.3). Assume that the turning operator $V$ satisfies the assumptions $\left(\boldsymbol{A}_{1}-\boldsymbol{A}_{2}-\boldsymbol{A}_{3}\right)$ and, when $\varepsilon \rightarrow 0$, the following statements hold true:

$$
\begin{aligned}
f_{\varepsilon} & \longrightarrow f \quad \text { a.e. in }[0, \infty) \times D_{\mathbf{x}} \times D_{\mathbf{v}} \times D_{u}, \\
\tilde{V}\left[f_{\varepsilon}\right] & \longrightarrow \tilde{V}[f]
\end{aligned}
$$

and the following quantities

$$
\left\langle f_{\varepsilon}\right\rangle, \quad\left\langle\mathbf{v} f_{\varepsilon}\right\rangle, \quad\left\langle\mathbf{v} \otimes \mathbf{v} f_{\varepsilon}\right\rangle, \quad\left\langle\tilde{J}\left[f_{\varepsilon}\right]\right\rangle, \quad\left\langle\tilde{N}\left[f_{\varepsilon}\right]\right\rangle,\left\langle\mathscr{T}_{F}\left[f_{\varepsilon}\right]\right\rangle,\left\langle\mathbf{v} \tilde{J}\left[f_{\varepsilon}\right]\right\rangle,\left\langle\mathbf{v} \mathscr{T}_{F}\left[f_{\varepsilon}\right]\right\rangle
$$

converge, in the sense of distributions on $\mathbb{R}_{+}^{*} \times D_{\mathbf{x}} \times D_{u}$, to the corresponding quantities

$$
\langle f\rangle, \quad\langle\mathbf{v} f\rangle, \quad\langle\mathbf{v} \otimes \mathbf{v} f\rangle, \quad\langle\tilde{J}[f]\rangle, \quad\langle\tilde{N}[f]\rangle, \quad\left\langle\mathscr{T}_{F}[f]\right\rangle, \quad\langle\mathbf{v} \tilde{J}[f]\rangle, \quad\left\langle\mathbf{v} \mathscr{T}_{F}[f]\right\rangle
$$

and that every formally small term in $\varepsilon$ vanishes. Then the local macroscopic density $\varrho$ is the weak solution of the following equation

$$
\partial_{t} \varrho+\delta_{\ell, 1} \partial_{u}(F(u)(1-u \mathbb{A}[\varrho](t)) \varrho)=\delta_{s, 1} \operatorname{div}_{\mathbf{x}}\left(\mathbb{D}_{\varrho} \cdot \nabla_{\mathbf{x}} \varrho\right)+\delta_{r, 1} H[\varrho]+\delta_{r+q, 1} S[\varrho],
$$

where

- $\mathbb{A}[\varrho](t)$ is the following operator:

$$
\mathbb{A}[\varrho](t)=\int_{D_{\mathbf{x}} \times D_{u}} u \varrho(t, \mathbf{x}, u) d \mathbf{x} d u,
$$

- $\mathbb{D}_{\varrho}$ is the following tensor:

$$
\mathbb{D}_{\varrho}=-\int_{D_{\mathbf{v}}} \mathbf{v} \otimes \chi(\mathbf{v}) d \mathbf{v}
$$


- $H[\varrho](t, \mathbf{x}, u)$ is the following operator:

$$
\begin{aligned}
H[\varrho] & =\langle G(\mathbf{v}), G(\mathbf{v})\rangle \int_{D_{u} \times D_{u}} \mathscr{A}\left(u_{*}, u^{*}, u\right) \varrho\left(t, \mathbf{x}, u_{*}\right) \varrho\left(t, \mathbf{x}, u^{*}\right) d u_{*} d u^{*} \\
& -\varrho(t, \mathbf{x}, u)\langle G(\mathbf{v}), G(\mathbf{v})\rangle \int_{D_{u}} \varrho\left(t, \mathbf{x}, u^{*}\right) d u^{*}
\end{aligned}
$$

- $S[\varrho](t, \mathbf{x}, u)$ is the following operator:

$$
S[\varrho]=\varrho(t, \mathbf{x}, u)\langle G(\mathbf{v}), G(\mathbf{v})\rangle \int_{D_{u}} \varrho\left(t, \mathbf{x}, u^{*}\right) d u^{*} .
$$

Proof. By taking the average of the equation (3.3) with respect to $\mathbf{v}$, using the assumption $\mathbf{A}_{1}$ and dividing by $\varepsilon$, one obtains:

$$
\partial_{t}\left\langle f_{\varepsilon}\right\rangle+\frac{1}{\varepsilon}\left\langle\mathbf{v} \cdot \nabla_{\mathbf{x}} f_{\varepsilon}\right\rangle+\varepsilon^{\ell-1}\left\langle\mathscr{T}_{F}\left[f_{\varepsilon}\right]\right\rangle=\varepsilon^{r-1}\left\langle\tilde{J}\left[f_{\varepsilon}\right]\right\rangle+\varepsilon^{r+q-1}\left\langle\tilde{N}\left[f_{\varepsilon}\right]\right\rangle .
$$

Assume that $\ell>1, r>1$, and $r+q>1$. When $\varepsilon \rightarrow 0$, the third, the fourth and the fifth terms in Eq. (3.31) goes to zero, therefore just the limit of the term:

$$
\frac{1}{\varepsilon}\left\langle\mathbf{v} \cdot \nabla_{\mathbf{x}} f_{\varepsilon}(t, \mathbf{x}, \mathbf{v}, u)\right\rangle
$$

has to be evaluated, see Lemma 3.2.

The term related to the Gaussian isokinetic thermostat reads:

$$
\begin{aligned}
\varepsilon^{\ell-1}\left\langle\mathscr{T}_{F}\left[f_{\varepsilon}\right]\right\rangle & =\varepsilon^{\ell-1}\left\langle\partial_{u}\left(F(u)\left(1-u \int_{\Omega} u f_{\varepsilon} d \mathbf{x} d \mathbf{v} d u\right) f_{\varepsilon}\right)\right\rangle \\
& \underset{\varepsilon \rightarrow 0}{\longrightarrow} \delta_{l, 1}\left\langle\partial_{u}\left(F(u)\left(1-u \int_{\Omega} u \varrho G d \mathbf{x} d \mathbf{v} d u\right) \varrho G\right)\right\rangle \\
& =\delta_{l, 1}\left\langle\partial_{u}\left(F(u)\left(1-u \int_{D_{\mathbf{x}} \times D_{u}} u \varrho d \mathbf{x} d u\right) \varrho G\right)\right\rangle \\
& =\delta_{l, 1} \partial_{u}(F(u)(1-u \mathbb{A}[\varrho](t)) \varrho) .
\end{aligned}
$$

Bearing all above in mind, it is an easy task to show that

$$
\varepsilon^{r-1}\left\langle\tilde{J}\left[f_{\varepsilon}\right]\right\rangle \underset{\varepsilon \rightarrow 0}{\longrightarrow} \delta_{r, 1} H[\varrho]
$$

and

$$
\varepsilon^{r+q-1}\left\langle\tilde{N}\left[f_{\varepsilon}\right]\right\rangle \underset{\varepsilon \rightarrow 0}{\longrightarrow} \delta_{r+q, 1} S[\varrho]
$$

where $H[\varrho]$ and $S[\varrho]$ are the operators defined in Eqs. (3.29) and (3.30), respectively. Therefore the proof is concluded.

Remark 4.1. In general the tensor $\mathbb{D}_{\varrho}$, called diffusivity tensor, is non-isotropic, i.e. is not a scalar multiple of identity.

Bearing all above in mind, the following theorem is related to the derivation of the macroscopic equation for the local macroscopic density of the $p$-thermostatted kinetic framework (2.9). 
Theorem 3.2. Let $f_{\varepsilon}(t, \mathbf{x}, \mathbf{v}, u)$ be a sequence of solutions of the scaled thermostatted kinetic equation (3.4). Assume that the turning operator $V$ satisfies the assumptions $\left(\boldsymbol{A}_{1}-\boldsymbol{A}_{2}-\boldsymbol{A}_{3}\right)$ and, when $\varepsilon \rightarrow 0$, the following statements hold true:

$$
\begin{aligned}
& f_{\varepsilon} \longrightarrow f \quad \text { a.e. in }[0, \infty) \times D_{\mathbf{x}} \times D_{\mathbf{v}} \times D_{u}, \\
& \tilde{V}\left[f_{\varepsilon}\right] \longrightarrow \tilde{V}[f]
\end{aligned}
$$

and the following quantities

$$
\left\langle f_{\varepsilon}\right\rangle, \quad\left\langle\mathbf{v} f_{\varepsilon}\right\rangle, \quad\left\langle\mathbf{v} \otimes \mathbf{v} f_{\varepsilon}\right\rangle, \quad\left\langle\tilde{J}\left[f_{\varepsilon}\right]\right\rangle, \quad\left\langle\tilde{N}\left[f_{\varepsilon}\right]\right\rangle,\left\langle\mathscr{T}_{F, p}\left[f_{\varepsilon}\right]\right\rangle,\left\langle\mathbf{v} \tilde{J}\left[f_{\varepsilon}\right]\right\rangle,\left\langle\mathbf{v} \mathscr{T}_{F, p}\left[f_{\varepsilon}\right]\right\rangle
$$

converge, in the sense of distributions on $\mathbb{R}_{+}^{*} \times D_{\mathbf{x}} \times D_{u}$, to the corresponding quantities

$$
\langle f\rangle, \quad\langle\mathbf{v} f\rangle, \quad\langle\mathbf{v} \otimes \mathbf{v} f\rangle, \quad\langle\tilde{J}[f]\rangle, \quad\langle\tilde{N}[f]\rangle, \quad\left\langle\mathscr{T}_{F, p}[f]\right\rangle, \quad\langle\mathbf{v} \tilde{J}[f]\rangle, \quad\left\langle\mathbf{v} \mathscr{T}_{F, p}[f]\right\rangle
$$

and that every formally small term in $\varepsilon$ vanishes. Then the local macroscopic density $\varrho$ is the weak solution of the following equation

$$
\partial_{t} \varrho+\delta_{\ell, 1} \partial_{u}\left(F(u)\left(1-u \mathbb{A}_{p}[\varrho](t)\right) \varrho\right)=\delta_{s, 1} \operatorname{div}_{\mathbf{x}}\left(\mathbb{D}_{\varrho} \cdot \nabla_{\mathbf{x}} \varrho\right)+\delta_{r, 1} H[\varrho]+\delta_{r+q, 1} S[\varrho],
$$

where $\mathbb{A}_{p}[\varrho](t)$ is the following operator:

$$
\mathbb{A}_{p}[\varrho](t)=\int_{D_{\mathbf{x}} \times D_{u}} u^{p-1} \varrho(t, \mathbf{x}, u) d \mathbf{x} d u,
$$

$\mathbb{D}_{\varrho}$ is the tensor (3.28), $H[\varrho]$ is the operator (3.29) and $S[\varrho]$ is the operator (3.30).

\section{Macroscopic equation for the relaxation model}

This section aims at showing the procedure of the asymptotic limit for a specific model: the relaxation model. In particular, since the directional changes are described by the kernel $T$, we can assume without restriction that the set of velocities $D_{\mathbf{v}}$ is symmetric with respect to the rotation group $\mathrm{SO}(d)$.

Specifically we assume that

$$
T\left(\mathbf{v}^{*}, \mathbf{v}\right)=\gamma G(\mathbf{v}), \quad 0<\gamma<\sigma .
$$

Therefore the turning operator reads:

$$
\begin{aligned}
V[f](t, \mathbf{x}, \mathbf{v}, u) & =\int_{D_{\mathbf{v}}}\left[T\left(\mathbf{v}^{*}, \mathbf{v}\right) f\left(t, \mathbf{x}, \mathbf{v}^{*}, u\right)-T\left(\mathbf{v}, \mathbf{v}^{*}\right) f(t, \mathbf{x}, \mathbf{v}, u)\right] d \mathbf{v}^{*} \\
& =\gamma\left(G(\mathbf{v}) \int_{D_{\mathbf{v}}} f\left(t, \mathbf{x}, \mathbf{v}^{*}, u\right) d \mathbf{v}^{*}-f(t, \mathbf{x}, \mathbf{v}, u) \int_{D_{\mathbf{v}}} G\left(\mathbf{v}^{*}\right) d \mathbf{v}^{*}\right) \\
& =\gamma(\varrho(t, \mathbf{x}, u) G(\mathbf{v})-f(t, \mathbf{x}, \mathbf{v}, u)) .
\end{aligned}
$$

Accordingly the function $\chi(\mathbf{v})$ solution of the integral equation $V[\chi(\mathbf{v})]=\mathbf{v} G(\mathbf{v})$ reads:

$$
\chi(\mathbf{v})=\frac{G(\mathbf{v})(\gamma \varrho-\mathbf{v})}{\gamma} .
$$


Consequently the diffusion tensor $\mathbb{D}_{\varrho}$ reads:

$$
\mathbb{D}_{\varrho}=-\int_{D_{\mathbf{v}}}[\mathbf{v} \otimes \chi(\mathbf{v})] d \mathbf{v}=\frac{1}{\gamma} \int_{D_{\mathbf{v}}}[\mathbf{v} \otimes \mathbf{v} G(\mathbf{v})] d \mathbf{v} .
$$

In particular if $D_{\mathbf{v}}$ is the 3 -sphere of radius $R>0$ and $G(\mathbf{v})=1 /\left|D_{\mathbf{v}}\right|$ we have

$$
\mathbb{D}_{\varrho}=\frac{1}{\gamma\left|D_{\mathbf{v}}\right|} \int_{D_{\mathbf{v}}} \mathbf{v} \otimes \mathbf{v} d \mathbf{v}
$$

By formula

$$
\int_{D_{\mathbf{v}}} v_{i} v_{j} d \mathbf{v}=\left|D_{\mathbf{v}}\right| \frac{R^{2}}{3} \delta_{i j}
$$

we have that

$$
\mathbb{D}_{\varrho}=\frac{R^{2}}{3 \gamma} \mathbb{I},
$$

where $\mathbb{I}$ is the identity matrix. Since

$$
\operatorname{div}_{\mathbf{x}}\left(\mathbb{D}_{\varrho} \cdot \nabla_{\mathbf{x}} \varrho\right)=\frac{R^{2}}{3 \gamma} \Delta_{\mathbf{x}} \varrho
$$

the macroscopic equations (3.26) and (3.36) thus read:

$$
\partial_{t} \varrho+\delta_{l, 1} \partial_{u}(F(u)(1-u \mathbb{A}[\varrho](t)) \varrho)=\delta_{s, 1} \frac{R^{2}}{3 \gamma} \Delta_{\mathbf{x}} \varrho+\delta_{r, 1} H[\varrho]+\delta_{r+q, 1} S[\varrho],
$$

and

$$
\partial_{t} \varrho+\delta_{l, 1} \partial_{u}\left(F(u)\left(1-u \mathbb{A}_{p}[\varrho](t)\right) \varrho\right)=\delta_{s, 1} \frac{R^{2}}{3 \gamma} \Delta_{\mathbf{x}} \varrho+\delta_{r, 1} H[\varrho]+\delta_{r+q, 1} S[\varrho],
$$

where $H[\varrho]$ and $S[\varrho]$ are defined by Eqs. (3.29) and (3.30), respectively, in which

$$
\langle G(\mathbf{v}), G(\mathbf{v})\rangle=\frac{1}{\left|D_{\mathbf{v}}\right|} .
$$

\section{The macroscopic framework by high-field scaling}

This section deals with the asymptotic limit of the thermostatted kinetic frameworks (2.1) and (2.9) by a high-field scaling. Specifically we consider the following scaling for the microscopic state variables and the macroscopic external force field:

$$
(t, \mathbf{x}, \mathbf{v}, u, F) \rightarrow\left(\frac{t}{\varepsilon}, \frac{\mathbf{x}}{\varepsilon}, \mathbf{v}, u, \varepsilon^{\ell} F\right), \quad l \geq 0,
$$

where $\varepsilon$ is a small real parameter.

Let $\mathscr{Z}$ be the total (initial) mass of the system

$$
\mathscr{Z}:=\int_{\Omega} f(0, \mathbf{x}, \mathbf{v}, u) d \mathbf{x} d \mathbf{v} d u,
$$

and $v_{0}$ the macroscopic mean velocity of the (initial) distribution

$$
v_{0}:=\frac{1}{\mathscr{Z}} \int_{\Omega} \mathbf{v} f(0, \mathbf{x}, \mathbf{v}, u) d \mathbf{x} d \mathbf{v} d u .
$$


Let $\tau$ be a typical time and $L$ a typical length of the system verifying the relation $\tau v_{0}=L$, so that $\tau$ and $L$ are the mechanical variables of the system, and $U$ a typical value of the activity. Then the dimensionless variables $t^{\prime}, \mathbf{x}^{\prime}, \mathbf{v}^{\prime}$, and $u^{\prime}$ are defined as follow:

$$
t:=\tau t^{\prime}, \quad \mathbf{x}:=L \mathbf{x}^{\prime}, \quad \mathbf{v}:=v_{0} \mathbf{v}^{\prime}, \quad u:=U u^{\prime} .
$$

Consequently the dimensionless distribution function reads:

$$
f^{\prime}\left(t^{\prime}, \mathbf{x}^{\prime}, \mathbf{v}^{\prime}, u^{\prime}\right):=\frac{L^{n} v_{0}^{n} U}{\mathscr{Z}} f(t, \mathbf{x}, \mathbf{v}, u) .
$$

Bearing all above in mind, the dimensionless thermostatted kinetic framework (2.1) thus reads:

$$
\partial_{t^{\prime}} f^{\prime}+\mathbf{v}^{\prime} \cdot \nabla_{\mathbf{x}^{\prime}} f^{\prime}+\mathscr{T}_{F}\left[f^{\prime}\right]=\frac{\tau \eta \mathscr{Z}}{L^{n} v_{0}^{n}}\left(\tilde{J}\left[f^{\prime}\right]+\mu \tilde{N}\left[f^{\prime}\right]\right)+\tau \nu \tilde{V}\left[f^{\prime}\right],
$$

Then the high-field scaling (5.1) is equivalent to the following choice

$$
\tau \nu=\frac{1}{\varepsilon}
$$

which means that the turning time (the inverse of the turning frequency $1 / \nu$ ) is small compared with the typical mechanical time of the system $\tau$. Removing the prime and rewriting Eq. (5.4), one has

$$
\left(\partial_{t}+\mathbf{v} \cdot \nabla_{\mathbf{x}}\right) f+\mathscr{T}_{F}[f]=\tau \frac{\eta \mathscr{Z}}{L^{n} v_{0}^{n}}(\tilde{J}[f]+\mu \tilde{N}[f])+\tau \nu \tilde{V}[f],
$$

According to [15], we assume the following relations

$$
\frac{\eta \mathscr{Z}}{L^{n} v_{0}^{n}}=\varepsilon^{r} \nu=\varepsilon^{r-1} \frac{1}{\tau}, \quad \mu=\varepsilon^{q},
$$

where $r \geq 1$ and $q \geq 0$. Then the scaled non-dimensional model (5.5) takes the form:

$$
\left(\partial_{t} f_{\varepsilon}+\mathbf{v} \cdot \nabla_{\mathbf{x}} f_{\varepsilon}\right)+\varepsilon^{\ell} \mathscr{T}_{F}\left[f_{\varepsilon}\right]=\tau \varepsilon^{r-1} \frac{1}{\tau}\left(\tilde{J}[f]+\varepsilon^{q} \tilde{N}[f]\right)+\frac{1}{\varepsilon} \tilde{V}[f],
$$

that can be rewritten as follows:

$$
\varepsilon\left(\partial_{t} f_{\varepsilon}+\mathbf{v} \cdot \nabla_{\mathbf{x}} f_{\varepsilon}\right)+\varepsilon^{\ell+1} \mathscr{T}_{F}\left[f_{\varepsilon}\right]=\varepsilon^{r} \tilde{J}\left[f_{\varepsilon}\right]+\varepsilon^{r+q} \tilde{N}\left[f_{\varepsilon}\right]+\tilde{V}\left[f_{\varepsilon}\right]
$$

and the scaled $p$-thermostatted framework (2.9) reads:

$$
\varepsilon\left(\partial_{t} f_{\varepsilon}+\mathbf{v} \cdot \nabla_{\mathbf{x}} f_{\varepsilon}\right)+\varepsilon^{\ell+1} \mathscr{T}_{F, p}\left[f_{\varepsilon}\right]=\varepsilon^{r} \tilde{J}\left[f_{\varepsilon}\right]+\varepsilon^{r+q} \tilde{N}\left[f_{\varepsilon}\right]+\tilde{V}\left[f_{\varepsilon}\right],
$$

where the meaning of each operator can be recovered by section 3.1 and where, as already mentioned in section 3.1, with a slight abuse of notation, we have set

$$
f_{\varepsilon}(t, \mathbf{x}, \mathbf{v}, u)=f\left(\frac{t}{\varepsilon}, \frac{\mathbf{x}}{\varepsilon}, \mathbf{v}, u\right) .
$$




\subsection{The macroscopic equations}

This subsection is concerned with the derivation of macroscopic equations for the local density and the first velocity momentum obtained by performing the asymptotic limit, under the highfield scaling, of the frameworks (5.7) and (5.8) as $\varepsilon$ goes to zero. The following lemma holds true.

Lemma 5.1. Let $f_{\varepsilon}(t, \mathbf{x}, \mathbf{v}, u)$ be a sequence of solutions of the scaled thermostatted kinetic equation (5.7) or (5.8). Assume that:

$\left(\boldsymbol{A}_{4}\right)$ For all $\varrho \in[0,+\infty)$ and $\mathbb{U} \in \mathbb{R}^{3}$ there exists a unique function

$$
F_{\varrho, \mathbb{U}}=F_{\varrho, \mathbb{U}}(\mathbf{v}) \in L^{1}\left(D_{\mathbf{v}},(1+|\mathbf{v}|) d \mathbf{v}\right)
$$

such that:

$$
V\left[F_{\varrho, \mathbb{U}}\right]=0, \quad \int_{D_{\mathbf{v}}} F_{\varrho, \mathbb{U}}(\mathbf{v}) d \mathbf{v}=\varrho, \quad \int_{D_{\mathbf{v}}} \mathbf{v} F_{\varrho, \mathbb{U}}(\mathbf{v}) d \mathbf{v}=\varrho \mathbb{U}
$$

$\left(\boldsymbol{A}_{5}\right)$ When $\varepsilon \rightarrow 0$, the following statements hold true:

$$
\begin{aligned}
& f_{\varepsilon} \longrightarrow f \quad \text { a.e. in }[0, \infty) \times D_{\mathbf{x}} \times D_{\mathbf{v}} \times D_{u}, \\
& \tilde{V}\left[f_{\varepsilon}\right] \longrightarrow \tilde{V}[f]
\end{aligned}
$$

and the following quantities

$$
\begin{gathered}
\left\langle f_{\varepsilon}\right\rangle,\left\langle\mathbf{v} f_{\varepsilon}\right\rangle,\left\langle\mathbf{v} \otimes \mathbf{v} f_{\varepsilon}\right\rangle,\left\langle\tilde{J}\left[f_{\varepsilon}\right]\right\rangle,\left\langle\mathscr{T}_{F}\left[f_{\varepsilon}\right]\right\rangle,\left\langle\mathscr{T}_{F, p}\left[f_{\varepsilon}\right]\right\rangle,\left\langle\tilde{N}\left[f_{\varepsilon}\right]\right\rangle, \\
\left\langle\mathbf{v} \tilde{J}\left[f_{\varepsilon}\right]\right\rangle,\left\langle\mathbf{v} \mathscr{T}_{F}\left[f_{\varepsilon}\right]\right\rangle,\left\langle\mathbf{v} \mathscr{T}_{F, p}\left[f_{\varepsilon}\right]\right\rangle,\left\langle\mathbf{v} \tilde{N}\left[f_{\varepsilon}\right]\right\rangle,
\end{gathered}
$$

converge, in the sense of distributions on $\mathbb{R}_{+}^{*} \times D_{\mathbf{x}} \times D_{u}$, to the corresponding quantities

$$
\begin{gathered}
\langle f\rangle, \quad\langle\mathbf{v} f\rangle, \quad\langle\mathbf{v} \otimes \mathbf{v} f\rangle, \quad\langle\tilde{J}[f]\rangle, \quad\left\langle\mathscr{T}_{F}[f]\right\rangle, \quad\left\langle\mathscr{T}_{F, p}[f]\right\rangle, \quad\langle\tilde{N}[f]\rangle, \\
\langle\mathbf{v} \tilde{J}[f]\rangle, \quad\left\langle\mathbf{v} \mathscr{T}_{F}[f]\right\rangle, \quad\left\langle\mathbf{v} \mathscr{T}_{F, p}[f]\right\rangle, \quad\langle\mathbf{v} \tilde{N}[f]\rangle,
\end{gathered}
$$

Then the asymptotic limit $f$ admits the following form

$$
f(t, \mathbf{x}, \mathbf{v}, u)=F_{\varrho(t, \mathbf{x}, u), \mathbb{U}(t, \mathbf{x}, u)}(\mathbf{v}) .
$$

Proof. Passing to the limit in Eq (5.7) or (5.8) when $\varepsilon \rightarrow 0$, yields $V\left[f_{0}\right]=0$. Setting

$$
\begin{aligned}
\varrho_{\varepsilon}(t, \mathbf{x}, u) & =\int_{D_{\mathbf{v}}} f_{\varepsilon}(t, \mathbf{x}, \mathbf{v}, u) d \mathbf{v}, \\
\varrho_{\varepsilon}(t, \mathbf{x}, u) \mathbb{U}_{\varepsilon}(t, \mathbf{x}, u) & =\int_{D_{\mathbf{v}}} \mathbf{v} f_{\varepsilon}(t, \mathbf{x}, \mathbf{v}, u) d \mathbf{v} .
\end{aligned}
$$

According to $\left(\mathbf{A}_{4}\right)$, there exists the unique function $F_{\varrho, \mathbb{U}}$, where $\mathbb{U}$ depends on $(t, \mathbf{x}, u)$, verifying the conditions (5.9). Therefore

$$
f_{0}(t, \mathbf{x}, \mathbf{v}, u)=F_{\varrho(t, \mathbf{x}, u), \mathbb{U}(t, \mathbf{x}, u)}(\mathbf{v}) .
$$


The main result of this section is the following theorem.

Theorem 5.1. Let $f_{\varepsilon}(t, \mathbf{x}, \mathbf{v}, u)$ be a sequence of solutions to the scaled thermostatted kinetic equation (5.7). Assume that $\left(\boldsymbol{A}_{1}-\boldsymbol{A}_{4}-\boldsymbol{A}_{5}\right)$ hold true and that every formally small term in $\varepsilon$ vanishes. Then $\varrho$ and $\varrho \mathbb{U}$ are the weak solutions of the following equations:

$$
\begin{gathered}
\partial_{t} \varrho+\operatorname{div}_{\mathbf{x}}(\varrho \mathbb{U})+\delta_{\ell, 0} K[\varrho]=\delta_{r, 1}\langle\tilde{J}[f]\rangle+\delta_{r+q, 1}\langle\tilde{N}[f]\rangle \\
\partial_{t}(\varrho \mathbb{U})+\operatorname{div}_{\mathbf{x}}(\varrho \mathbb{U} \otimes \mathbb{U}+\mathbb{P})+\delta_{\ell, 0} S[\varrho \mathbb{U}]=\delta_{r, 1}\langle\mathbf{v} \tilde{J}[f]\rangle+\delta_{r+q, 1}\langle\mathbf{v} \tilde{N}[f]\rangle
\end{gathered}
$$

where

$$
\begin{aligned}
K[\varrho] & =\partial_{u}(F(u)(1-u \mathbb{A}[\varrho](t)) \varrho) \\
S[\varrho \mathbb{U}] & =\partial_{u}(F(u)(1-u \mathbb{A}[\varrho](t)) \varrho \mathbb{U})
\end{aligned}
$$

being $\mathbb{P}$ the pressure tensor (2.13).

Proof. According to Lemma 5.1 we can introduce the function $g$ (uniformly bounded in a suitable functional space) such that:

$$
f_{\varepsilon}(t, \mathbf{x}, \mathbf{v}, u)=F_{\varrho, \mathbb{U}}(\mathbf{v})+\varepsilon g(t, \mathbf{x}, \mathbf{v}, u) .
$$

Let $\psi \in\{1, \mathbf{v}\}$. Then multiplying the scaled Eq. (5.7) by $\psi$ and integrating with respect to velocity variable $\mathbf{v}$ we have

$$
\begin{aligned}
\varepsilon \int_{D_{\mathbf{v}}}\left(\partial_{t}\right. & \left.+\mathbf{v} \cdot \nabla_{\mathbf{x}}\right) f_{\varepsilon} \psi d \mathbf{v}+\varepsilon^{\ell+1} \partial_{u} \int_{D_{\mathbf{v}}} \psi \mathscr{T}_{F}\left[f_{\varepsilon}\right] d \mathbf{v} \\
& =\varepsilon^{r} \int_{D_{\mathbf{v}}} \psi \tilde{J}\left[f_{\varepsilon}\right] d \mathbf{v}+\varepsilon^{r+q} \int_{D_{\mathbf{v}}} \psi \tilde{N}\left[f_{\varepsilon}\right] d \mathbf{v}+\int_{D_{\mathbf{v}}} \psi \tilde{V}\left[f_{\varepsilon}\right] d \mathbf{v} .
\end{aligned}
$$

Therefore we have

$$
\begin{gathered}
\partial_{t}\left\langle f_{\varepsilon}\right\rangle+\left\langle\mathbf{v} \cdot \nabla_{\mathbf{x}} f_{\varepsilon}\right\rangle+\varepsilon^{\ell}\left\langle\partial_{u} \mathscr{T}_{F}\left[f_{\varepsilon}\right]\right\rangle=\varepsilon^{r-1}\left\langle\tilde{J}\left[f_{\varepsilon}\right]\right\rangle+\varepsilon^{r+q-1}\left\langle\tilde{N}\left[f_{\varepsilon}\right]\right\rangle, \\
\partial_{t}\left\langle\mathbf{v} f_{\varepsilon}\right\rangle+\operatorname{div}_{\mathbf{x}}\left\langle\mathbf{v} \otimes \mathbf{v} f_{\varepsilon}\right\rangle+\varepsilon^{\ell}\left\langle\mathbf{v} \partial_{u} \mathscr{T}_{F}\left[f_{\varepsilon}\right]\right\rangle=\varepsilon^{r-1}\left\langle\mathbf{v} \tilde{J}\left[f_{\varepsilon}\right]\right\rangle+\varepsilon^{r+q-1}\left\langle\mathbf{v} \tilde{N}\left[f_{\varepsilon}\right]\right\rangle .
\end{gathered}
$$

Then

$$
\begin{aligned}
& \partial_{t}\left\langle f_{\varepsilon}\right\rangle+\left\langle\mathbf{v} \cdot \nabla_{\mathbf{x}} f_{\varepsilon}\right\rangle \longrightarrow \partial_{t} \varrho+\operatorname{div}_{\mathbf{x}}(\varrho \mathbb{U}) \\
& \partial_{t}\left\langle\mathbf{v} f_{\varepsilon}\right\rangle+\operatorname{div}_{\mathbf{x}}\left\langle\mathbf{v} \otimes \mathbf{v} f_{\varepsilon}\right\rangle \longrightarrow \partial_{t}(\varrho \mathbb{U})+\nabla_{\mathbf{x}} \cdot\left(\int_{D_{\mathbf{v}}} \mathbf{v} \otimes \mathbf{v} F_{\varrho, \mathbb{U}}\right) .
\end{aligned}
$$

Since

$$
\int(\mathbf{v} \otimes \mathbf{v}) F_{\varrho, \mathbb{U}} d \mathbf{v}=\varrho \mathbb{U} \otimes \mathbb{U}+\mathbb{P},
$$

we have

$$
\partial_{t}\left\langle\mathbf{v} f_{\varepsilon}\right\rangle+\operatorname{div}_{\mathbf{x}}\left\langle\mathbf{v} \otimes \mathbf{v} f_{\varepsilon}\right\rangle \longrightarrow \partial_{t}(\varrho \mathbb{U})+\nabla_{\mathbf{x}} \cdot(\varrho \mathbb{U} \otimes \mathbb{U}+\mathbb{P}) .
$$

The velocity average of the thermostatted term reads:

$$
\left\langle\partial_{u} \mathscr{T}_{F}\left[f_{\varepsilon}\right]\right\rangle=\left\langle\partial_{u}\left(F(u)\left(1-u \int_{\Omega} u f_{\varepsilon} d \mathbf{x} d \mathbf{v} d u\right) f_{\varepsilon}\right)\right\rangle .
$$


Letting $\varepsilon$ go to zero, and according to the (5.12), we have:

$$
\begin{aligned}
\left\langle\partial_{u} \mathscr{T}_{F}\left[f_{\varepsilon}\right]\right\rangle & \underset{\varepsilon \rightarrow 0}{\longrightarrow} \quad K[\varrho]=\left\langle\partial_{u}\left(F(u)\left(1-u \int_{\Omega} u F_{\varrho, \mathbb{U}} d \mathbf{x} d \mathbf{v} d u\right) F_{\varrho, \mathbb{U}}\right)\right\rangle \\
& =\left\langle\partial_{u}\left(F(u)\left(1-u \int_{D_{\mathbf{x}} \times D_{u}} u \varrho d \mathbf{x} d u\right) F_{\varrho, \mathbb{U}}\right)\right\rangle . \\
& =\partial_{u}(F(u)(1-u \mathbb{A}[\varrho](t)) \varrho),
\end{aligned}
$$

where $\mathbb{A}[\varrho]$ is given by Eq. (3.27). Moreover

$$
\left\langle\mathbf{v} \partial_{u} \mathscr{T}_{F}\left[f_{\varepsilon}\right]\right\rangle=\left\langle\mathbf{v} \partial_{u}\left(F(u)\left(1-u \int_{\Omega} u f_{\varepsilon} d \mathbf{x} d \mathbf{v} d u\right) f_{\varepsilon}\right)\right\rangle,
$$

then

$$
\begin{aligned}
\left\langle\mathbf{v} \partial_{u} \mathscr{T}_{F}\left[f_{\varepsilon}\right]\right\rangle & \underset{\varepsilon \rightarrow 0}{\longrightarrow} \quad S[\varrho \mathbb{U}]=\left\langle\mathbf{v} \partial_{u}\left(F(u)\left(1-u \int_{\Omega} u F_{\varrho, \mathbb{U}} d \mathbf{x} d \mathbf{v} d u\right) F_{\varrho, \mathbb{U}}\right)\right\rangle \\
& =\left\langle\partial_{u}\left(F(u)\left(1-u \int_{D_{\mathbf{x}} \times D_{u}} u \varrho d \mathbf{x} d u\right) \mathbf{v} F_{\varrho, \mathbb{U}}\right)\right\rangle \\
& =\partial_{u}(F(u)(1-u \mathbb{A}[\varrho](t)) \varrho \mathbb{U}) .
\end{aligned}
$$

Therefore the proof is concluded.

Bearing all above in mind, the following theorem is related to the derivation of the macroscopic equations for the local density and the first velocity momentum of the $p$-thermostatted kinetic framework (2.9).

Theorem 5.2. Let $f_{\varepsilon}(t, \mathbf{x}, \mathbf{v}, u)$ be a sequence of solutions to the scaled thermostatted kinetic equation (5.8). Assume that $\left(\boldsymbol{A}_{1}-\boldsymbol{A}_{4}-\boldsymbol{A}_{5}\right)$ hold true and that every formally small term in $\varepsilon$ vanishes. Then $\varrho$ and $\varrho \mathbb{U}$ are the weak solutions of the following equations:

$$
\begin{gathered}
\partial_{t} \varrho+\operatorname{div}_{\mathbf{x}}(\varrho \mathbb{U})+\delta_{\ell, 0} K_{p}[\varrho]=\delta_{r, 1}\langle\tilde{J}[f]\rangle+\delta_{r+q, 1}\langle\tilde{N}[f]\rangle \\
\partial_{t}(\varrho \mathbb{U})+\operatorname{div}_{\mathbf{x}}(\varrho \mathbb{U} \otimes \mathbb{U}+\mathbb{P})+\delta_{\ell, 0} S_{p}[\varrho \mathbb{U}]=\delta_{r, 1}\langle\mathbf{v} \tilde{J}[f]\rangle+\delta_{r+q, 1}\langle\mathbf{v} \tilde{N}[f]\rangle
\end{gathered}
$$

where

$$
\begin{aligned}
K_{p}[\varrho] & =\partial_{u}\left(F(u)\left(1-u \mathbb{A}_{p}[\varrho](t)\right) \varrho\right) \\
S_{p}[\varrho \mathbb{U}] & =\partial_{u}\left(F(u)\left(1-u \mathbb{A}_{p}[\varrho](t)\right) \varrho \mathbb{U}\right)
\end{aligned}
$$

being $\mathbb{A}_{p}[\varrho](t)$ is the operator (3.37).

\subsection{Macroscopic equations for the relaxation model}

In this subsection we assume that the velocity domain $D_{\mathbf{v}}$ is the 2 -sphere of radius $R>0$. We assume as turning kernel the following function:

$$
T\left(\mathbf{v}, \mathbf{v}^{*}\right)=\frac{1}{\left|D_{\mathbf{v}}\right|}\left(1+\frac{a}{R^{2}}\left(\mathbf{v} \cdot \mathbf{v}^{*}\right)\right) \quad \text { with } \quad a<3 .
$$

Therefore the turning operator reads:

$$
V[f]=\frac{\varrho}{\left|D_{\mathbf{v}}\right|}\left(1+\frac{a}{R^{2}} \mathbf{v} \cdot \mathbb{U}\right)-f(\mathbf{v}) .
$$


Moreover we have

$$
F_{\varrho, \mathbb{U}}(\mathbf{v})=\frac{\varrho}{\left|D_{\mathbf{v}}\right|}\left(1+\frac{a}{R^{2}} \mathbf{v} \cdot \mathbb{U}\right) .
$$

Let $u^{*}, u_{*} \in D_{u}$ and $\varrho^{*}=\varrho\left(t, \mathbf{x}, u^{*}\right), \mathbb{U}^{*}=\mathbb{U}\left(t, \mathbf{x}, u^{*}\right), \varrho_{*}=\varrho\left(t, \mathbf{x}, u_{*}\right), \mathbb{U}_{*}=\mathbb{U}\left(t, \mathbf{x}, u_{*}\right)$ and

$$
F_{\varrho_{*}, \mathbb{U}_{*}}(\mathbf{v})=\frac{\varrho_{*}}{\left|D_{\mathbf{v}}\right|}\left(1+\frac{a}{R^{2}} \mathbf{v} \cdot \mathbb{U}_{*}\right), \quad F_{\varrho^{*}, \mathbb{U}^{*}}(\mathbf{v})=\frac{\varrho^{*}}{\left|D_{\mathbf{v}}\right|}\left(1+\frac{a}{R^{2}} \mathbf{v} \cdot \mathbb{U}^{*}\right)
$$

then by straightforward computations we have

$$
\int_{D_{\mathbf{v}}} F_{\varrho_{*}, \mathbb{U}_{*}}(\mathbf{v}) F_{\varrho^{*}, \mathbb{U}^{*}}(\mathbf{v}) d \mathbf{v}=\frac{\varrho_{*} \varrho^{*}}{\left|D_{\mathbf{v}}\right|}\left(1+\frac{a^{2}}{R^{2}} \mathbb{U}_{*} \cdot \mathbb{U}^{*}\right),
$$

and

$$
\int_{D_{\mathbf{v}}} \mathbf{v} F_{\varrho_{*}, \mathbb{U}_{*}}(\mathbf{v}) F_{\varrho^{*}, U^{*}}(\mathbf{v}) d \mathbf{v}=\frac{\varrho_{*} \varrho^{*}}{\left|D_{\mathbf{v}}\right|} \frac{a^{2}}{3 R^{2}}\left(\mathbb{U}_{*}+\mathbb{U}^{*}\right) .
$$

Finally, If $f=F_{\varrho, \mathbb{U}}$, where $F_{\varrho, \mathbb{U}}$ is given by Eq. (5.30), straightforward computations show:

$$
\begin{gathered}
\int_{D_{\mathbf{v}}} \tilde{J}[f] d \mathbf{v}=\frac{1}{\left|D_{\mathbf{v}}\right|}\left(J[\varrho]+\frac{a^{2}}{3 R^{2}} J[\varrho \mathbb{U}]\right), \\
\int_{D_{\mathbf{v}}} \mathbf{v} \tilde{J}[f] d \mathbf{v}=\frac{a^{2}}{3 R^{2}\left|D_{\mathbf{v}}\right|}(A[\varrho, \varrho \mathbb{U}]+B[\varrho, \varrho \mathbb{U}]), \\
\int_{D_{\mathbf{v}}} \tilde{N}[f] d \mathbf{v}=\frac{1}{\left|D_{\mathbf{v}}\right|}\left(N[\varrho]+\frac{a^{2}}{3 R^{2}} N[\varrho \mathbb{U}]\right), \\
\int_{D_{\mathbf{v}}} \mathbf{v} \tilde{N}[f] d \mathbf{v}=\frac{a^{2}}{3 R^{2}\left|D_{\mathbf{v}}\right|}(C[\varrho, \varrho \mathbb{U}]+D[\varrho, \varrho \mathbb{U}]),
\end{gathered}
$$

where

$$
\begin{gathered}
A[\varrho, \varrho \mathbb{U}])=\int_{D_{u} \times D_{u}} \mathscr{A}\left(u_{*}, u^{*}, u\right) \varrho_{*} \mathbb{U}_{*} \varrho^{*} d u_{*} d u^{*}-\varrho \mathbb{U} \int_{D_{u} \times D_{u}} \varrho^{*} d u^{*}, \\
B[\varrho, \varrho \mathbb{U}]=\int_{D_{u} \times D_{u}} \mathscr{A}\left(u_{*}, u^{*}, u\right) \varrho^{*} \mathbb{U}^{*} \varrho_{*} d u_{*} d u^{*}-\varrho \int_{D_{u} \times D_{u}} \varrho^{*} \mathbb{U}^{*} d u^{*} . \\
C[\varrho, \varrho \mathbb{U}]=\varrho \mathbb{U} \int_{D_{u} \times D_{u}} \varrho^{*} d u^{*}, \\
D[\varrho, \varrho \mathbb{U}]=\int_{D_{u} \times D_{u}} \varrho^{*} \mathbb{U}^{*} d u^{*} .
\end{gathered}
$$

Bearing all above in mind and assuming that for $\psi \in\{1, \mathbf{v}\}$ the moments

$$
\left\langle\psi f_{\varepsilon}\right\rangle, \quad\left\langle\psi J\left[\mathbf{f}_{\varepsilon}\right]\right\rangle, \quad\left\langle\psi \mathscr{T}_{F}\left[f_{\varepsilon}\right]\right\rangle, \quad\left\langle\psi \mathscr{T}_{F, p}\left[f_{\varepsilon}\right]\right\rangle,
$$

converge in the sense of distributions to the corresponding moments

$$
\langle\psi f\rangle, \quad\langle\psi J[\mathbf{f}]\rangle, \quad\left\langle\psi \mathscr{T}_{F}[f]\right\rangle, \quad\left\langle\psi \mathscr{T}_{F, p}[f]\right\rangle,
$$


then the macroscopic systems (5.16)-(5.17) and (5.27)-(5.28) thus reads:

$$
\left\{\begin{array}{r}
\partial_{t} \varrho+\operatorname{div}_{\mathbf{x}}(\varrho \mathbb{U})+\delta_{l, 0} \partial_{u}(F(u)(1-u \mathbb{A}[\varrho](t)) \varrho) \\
=\frac{\delta_{r, 1}}{\left|D_{\mathbf{v}}\right|}\left(J[\varrho]+\frac{a^{2}}{3 R^{2}} J[\varrho \mathbb{U}]\right)+\frac{\delta_{r+q, 1}}{\left|D_{\mathbf{v}}\right|}\left(N[\varrho]+\frac{a^{2}}{3 R^{2}} N[\varrho \mathbb{U}]\right), \\
\partial_{t}(\varrho \mathbb{U})+\frac{R^{2}}{3} \nabla_{\mathbf{x}} \varrho+\delta_{l, 0} \partial_{u}(F(u)(1-u \mathbb{A}[\varrho](t)) \varrho \mathbb{U}) \\
=\frac{a^{2} \delta_{r, 1}}{3 R^{2}\left|D_{\mathbf{v}}\right|}(A[\varrho, \varrho \mathbb{U}]+B[\varrho, \varrho \mathbb{U}])+\frac{a^{2} \delta_{r+q, 1}}{3 R^{2}\left|D_{\mathbf{v}}\right|}(C[\varrho, \varrho \mathbb{U}]+D[\varrho, \varrho \mathbb{U}]),
\end{array}\right.
$$

and

$$
\left\{\begin{array}{r}
\partial_{t} \varrho+\operatorname{div}_{\mathbf{x}}(\varrho \mathbb{U})+\delta_{l, 0} \partial_{u}\left(F(u)\left(1-u \mathbb{A}_{p}[\varrho](t)\right) \varrho\right) \\
=\frac{\delta_{r, 1}}{\left|D_{\mathbf{v}}\right|}\left(J[\varrho]+\frac{a^{2}}{3 R^{2}} J[\varrho \mathbb{U}]\right)+\frac{\delta_{r+q, 1}}{\left|D_{\mathbf{v}}\right|}\left(N[\varrho]+\frac{a^{2}}{3 R^{2}} N[\varrho \mathbb{U}]\right), \\
\partial_{t}(\varrho \mathbb{U})+\frac{R^{2}}{3} \nabla_{\mathbf{x}} \varrho+\delta_{l, 0} \partial_{u}\left(F(u)\left(1-u \mathbb{A}_{p}[\varrho](t)\right) \varrho \mathbb{U}\right) \\
=\frac{a^{2} \delta_{r, 1}}{3 R^{2}\left|D_{\mathbf{v}}\right|}(A[\varrho, \varrho \mathbb{U}]+B[\varrho, \varrho \mathbb{U}])+\frac{a^{2} \delta_{r+q, 1}}{3 R^{2}\left|D_{\mathbf{v}}\right|}(C[\varrho, \varrho \mathbb{U}]+D[\varrho, \varrho \mathbb{U}]) .
\end{array}\right.
$$

\section{$6 \quad$ Further generalizations and future research directions}

The topic of the present paper has been the derivation of macroscopic equations for the local density and first velocity momentum of the thermostatted kinetic frameworks which model systems whose particles have the ability to perform some strategies, such as the changing into the activity variable of the microscopic state, proliferation or destruction of particles. The thermostatted frameworks include conservative and nonconservative interactions and a velocity-jump process; the space variable is not modified by the interactions.

The main interest of this paper has been focused on the derivation of macroscopic equations by asymptotic methods consisting in passing to the limit of the scaled framework for vanishing mean free path. Moreover an integral approach has been used by employing test functions directly on conservation equations for the velocity moments of distribution function and balancing with care the various terms. The main results have been obtained by considering some assumptions on the turning operators on which the asymptotic method is based.

A specific model, namely the relaxation model, has been also discussed into the paper with the aim of showing in more details the asymptotic method and its applications.

According to our results, see equations (3.26) and (3.36), and differently from the pertinent literature, see, among others, the review paper [39], two convective terms appear into the macroscopic equation. Moreover, depending on the scaling, nonconservative interactions can contribute to the time evolution of the local macroscopic density of the system under consideration. It is easy to see that, when $s>1$, the macroscopic equations (3.26) and (3.36) read

$$
\partial_{t} \varrho+\delta_{l, 1} \partial_{u}(F(u)(1-u \mathbb{A}[\varrho](t)) \varrho)=\delta_{r, 1} H[\varrho]+\delta_{r+q, 1} S[\varrho],
$$

and

$$
\partial_{t} \varrho+\delta_{l, 1} \partial_{u}\left(F(u)\left(1-u \mathbb{A}_{p}[\varrho](t)\right) \varrho\right)=\delta_{r, 1} H[\varrho]+\delta_{r+q, 1} S[\varrho],
$$


therefore the diffusion time scale $\varepsilon t$ does not contribute to the diffusion approximation. In this case, one proposes the following scalings:

$$
\varepsilon\left(\partial_{t}+\mathbf{v} \cdot \nabla_{\mathbf{x}}\right) f_{\varepsilon}+\varepsilon^{l} \mathscr{T}_{F}[f]=\varepsilon^{r} \tilde{J}\left[f_{\varepsilon}\right]+\varepsilon^{r+q} \tilde{N}\left[f_{\varepsilon}\right]+\tilde{V}\left[f_{\varepsilon}\right],
$$

and

$$
\varepsilon\left(\partial_{t}+\mathbf{v} \cdot \nabla_{\mathbf{x}}\right) f_{\varepsilon}+\varepsilon^{l} \mathscr{T}_{F, p}[f]=\varepsilon^{r} \tilde{J}\left[f_{\varepsilon}\right]+\varepsilon^{r+q} \tilde{N}\left[f_{\varepsilon}\right]+\tilde{V}\left[f_{\varepsilon}\right]
$$

which give the asymptotic equations (6.1) and (6.2).

The mathematical framework proposed in the present paper refers to the modeling of biological and chemical systems whose description is based on the definition of a distribution function only. However according to a new system biology approach, see papers [25], it is often necessary to consider different populations of particles characterized by a specific strategy. According to this system biology approach, the whole system is decomposed into a finite number $n$ of functional subsystems whose distribution functions are denoted by $f_{i}=f_{i}(t, u)$, for $i \in\{1,2, \ldots, n\}$, and the thermostatted kinetic framework now reads:

$$
\left(\partial_{t}+\mathbf{v} \cdot \nabla_{\mathbf{x}}\right) f_{i}+\partial_{u}\left(F_{i}(u)\left(1-u \int_{\Omega} u \tilde{f} d \mathbf{x} d \mathbf{v} d u\right) f_{i}\right)=J_{i}[\mathbf{f}]+N_{i}[\mathbf{f}]+\nu V_{i}\left[f_{i}\right],
$$

where $\mathbf{f}=\left(f_{1}, f_{2}, \ldots, f_{n}\right)$ is the vector whose components are the distribution functions of the functional subsystems,

$$
\tilde{f}(t, \mathbf{x}, \mathbf{v}, u)=\sum_{i=1}^{n} f_{i}(t, \mathbf{x}, \mathbf{v}, u)
$$

and the conservative, nonconservative and velocity-jump operators now read:

$$
\begin{aligned}
G_{i}[\mathbf{f}] & =\sum_{j=1}^{n} \int_{D_{u} \times D_{u}} \eta_{i j}\left(u_{*}, u^{*}\right) \mathscr{A}_{i j}\left(u_{*}, u^{*}, u\right) f_{i}\left(t, \mathbf{x}, \mathbf{v}, u_{*}\right) f_{j}\left(t, \mathbf{x}, \mathbf{v}, u^{*}\right) d u_{*} d u^{*} \\
L_{i}[\mathbf{f}] & =-f_{i}(t, \mathbf{x}, \mathbf{v}, u) \sum_{j=1}^{n} \int_{D_{u}} \eta_{i j}\left(u_{*}, u^{*}\right) f_{j}\left(t, \mathbf{x}, \mathbf{v}, u^{*}\right) d u^{*}
\end{aligned}
$$

and

$$
\begin{gathered}
N_{i}[\mathbf{f}]=f_{i}(t, \mathbf{x}, \mathbf{v}, u) \sum_{j=1}^{n} \int_{D_{u}} \eta_{i j}\left(u_{*}, u^{*}\right) \mu_{i j}\left(u_{*}, u^{*}\right) f_{j}\left(t, \mathbf{x}, \mathbf{v}, u^{*}\right) d u^{*}, \\
V_{i}\left[f_{i}\right]=\int_{D_{\mathbf{v}}}\left[T_{i}\left(\mathbf{v}^{*}, \mathbf{v}\right) f_{i}\left(t, \mathbf{x}, \mathbf{v}^{*}, u\right)-T_{i}\left(\mathbf{v}, \mathbf{v}^{*}\right) f_{i}(t, \mathbf{x}, \mathbf{v}, u)\right] d \mathbf{v}^{*},
\end{gathered}
$$

where the meaning of each term into the operators can be recovered from the previous sections. The asymptotic methods performed into the present paper can be applied also for the above framework (6.5) and in particular, in the case of the low-field limit, we obtain the following macroscopic equation:

$$
\partial_{t} \varrho_{i}+\delta_{\ell, 1} \partial_{u}\left(F(u)(1-u \tilde{\mathbb{A}}[\varrho](t)) \varrho_{i}\right)=\delta_{s, 1} \operatorname{div}_{\mathbf{x}}\left(\mathbb{D}_{\varrho_{i}} \cdot \nabla_{\mathbf{x}} \varrho_{i}\right)+\delta_{r, 1} H_{i}[\varrho]+\delta_{r+q, 1} S_{i}[\varrho],
$$

where $\varrho=\left(\varrho_{1}, \varrho_{2}, \ldots, \varrho_{n}\right)$

$$
\tilde{\mathbb{A}}[\varrho](t)=\sum_{j=1}^{n} \int_{D_{\mathbf{x}} \times D_{u}} u \varrho_{j}(t, \mathbf{x}, u) d \mathbf{x} d u
$$


$\mathbb{D}_{\varrho_{i}}$ is the following tensor:

$$
\mathbb{D}_{\varrho_{i}}=-\int_{D_{\mathbf{v}}} \mathbf{v} \otimes \chi_{i}(\mathbf{v}) d \mathbf{v}
$$

$H_{i}[\varrho](t)$ is the following operator:

$$
\begin{aligned}
H_{i}[\varrho] & =\sum_{j=1}^{n}\left\langle G_{i}(\mathbf{v}), G_{j}(\mathbf{v})\right\rangle \int_{D_{u} \times D_{u}} \mathscr{A}_{i j}\left(u_{*}, u^{*}, u\right) \varrho_{i}\left(t, \mathbf{x}, u_{*}\right) \varrho_{j}\left(t, \mathbf{x}, u^{*}\right) d u_{*} d u^{*} \\
& -\varrho_{i}(t, \mathbf{x}, u) \sum_{j=1}^{n}\left\langle G_{i}(\mathbf{v}), G_{j}(\mathbf{v})\right\rangle \int_{D_{u}} \varrho_{j}\left(t, \mathbf{x}, u^{*}\right) d u^{*} .
\end{aligned}
$$

and $S_{i}[\varrho](t)$ is the following operator:

$$
S_{i}[\varrho]=\varrho_{i}(t, \mathbf{x}, u) \sum_{j=1}^{n}\left\langle G_{i}(\mathbf{v}), G_{j}(\mathbf{v})\right\rangle \int_{D_{u}} \varrho_{j}\left(t, \mathbf{x}, u^{*}\right) d u^{*} .
$$

Similar arguments hold true also for the high-field limit whose generalization is technical.

It is worth stressing that the choice of the scaling plays an important role in the derivation of macroscopic equations and specifically, future research directions include the possibility to develop a mixture of scalings (high-low field) that can be able to describe most phenomena occurring in complex chemical systems.

Another research perspective refers to the asymptotic analysis of thermostatted kinetic frameworks which include external actions at the microscopic scale (open systems) for the modeling of the surrounding environment. This work is in progress and results will be presented in due course.

Further developments of the asymptotic analysis performed in this paper refer to the derivation of the macroscopic equation by the low-field and high-field scalings for the thermostatted kinetic framework (2.1) coupled with the mutative operator, see [40], which takes into account mutations occurring in biological and chemical systems.

The asymptotic analysis developed into the present paper is particularly important for the multiscale analysis of complex biological and chemical systems which definitely represents an hard challenge.

\section{Acknowledgement}

CB and AL were partially supported by L'Agence Nationale de la Recherche (ANR T-KiNeT Project).

\section{References}

[1] R. Eftimie, J.L. Bramson, D.J.D. Earn, Interactions between the immune system and cancer: a brief review of non-spatial mathematical models, Bulletin of Mathematical Biology 73 (2011) $2-32$.

[2] C. Bianca, Bellomo N., Towards a Mathematical Theory of Multiscale Complex Biological Systems. World Scientific, Series in Mathematical Biology and Medicine, 2011.

[3] C. Bianca, Thermostatted kinetic equations as models for complex systems in physics and life sciences, Physics of Life Reviews Vol. 9, Issue 4, 2012, pp. 359-399. 
[4] A. Chauviere, L. Preziosi, and C. Verdier, Eds., Cell Mechanics, CRC Press, Boca Raton, (2010).

[5] L. F. Henderson, On the fluid mechanic of human crowd motion, Transp Research 8 (1975) 509-515.

[6] R. L. Hughes, The flow of human crowds, Annual Reviews of Fluid Mechanics 35 (2003) 169-183.

[7] C. Bardos, F. Golse and D. Levermore, Fluid dynamic limits of kinetic equations I, Formal derivations, J. Statist. Phys. 21 (1992) 531-555.

[8] P. L. Lions and N. Masmoudi, From Boltzmann equation to the equation of incompressible fluid mechanics - I, Arch. Rational. Mech. Anal. 158 (2001) 173-193.

[9] F. Filbet, P. Laurencot, B. Perthame, Derivation of hyperbolic models for chemosensitive movement, J Math Biol. 50 (2005) 189-207.

[10] C. Dogbe, Fluid dynamic limits for gas mixture. I. Formal derivations. Math. Models Methods Appl. Sci. 18 (2008), no. 9, 1633-1672.

[11] T. Hillen and H. Othmer, The diffusion limit of transport equations derived from velocity jump processes, SIAM J. Appl. Math. 61 (2000) 751-775.

[12] H. G. Othmer, S. R. Dunbar, W. Alt, Models of dispersal in biological systems, J. Math. Biol. 26 (1988) 263-298.

[13] T. Goudon, O. Sánchez, J. Soler and L. L. Bonilla, Low-field limit for a nonlinear discrete driftdiffusion model arising in semiconductor superlattices theory, SIAM J. Appl. Math. 64 (2004) 1526-1549.

[14] C. Villani, A review of mathematical problems in collisional kinetic theory, Handbook of Mathematical Fluid Dynamics, Vol. II, Friedlander, S. and Serre, D., eds., North-Holland, Amsterdam (2003).

[15] F. Poupaud, Runaway phenomena and fluid approximation under high-fields in semiconductor kinetic theory, Z. Angew. Math. Mech. 72 (1992) 359-372.

[16] W. Alt, Biased random walk models for chemotaxis and related diffusion approximations. J. Math. Biol., 9:147-177, 1980.

[17] G. C. Papanicolaou, Asymptotic analysis of transport processes. Bulletin AMS, 81:330-392, 1975.

[18] A. Bellouquid and C. Bianca, Modelling aggregation-fragmentation phenomena from kinetic to macroscopic scales, Math. Comput. Modelling 52 (2010) 802-813.

[19] P. Degond and B. Wennberg, Mass and energy balance laws derived from high-field limits of thermostatted Boltzmann equations, Commun Math Sci. 5 (2007) 355-382.

[20] H. Grad, Asymptotic theory of the Boltzmann equation. In "Rarefied Gas Dynamics" (Proc. 3rd Internat. Sympos., Palais de l'UNESCO, Paris 1962) Vol. 1, 26-59.

[21] C. Cercignani, The Boltzmann equation and its applications, New York: Springer-Verlag, 1988.

[22] F. Golse, C.D. Levermore, Hydrodynamic limits of kinetic models, in: T. Passot et al. (Eds.), Topics in Kinetic Theory, in: Fields Institute Communications, vol. 46, Providence, 2005.

[23] Mellet, Fractional diffusion limit for collisional kinetic equations: a Hilbert expansion approach, Kinet. Relat. Models 4 (2011), no. 4, 873-900.

[24] C. Bianca, M. Ferrara, L. Guerrini, High-order moments conservation in thermostatted kinetic models, J Glob Optim 58 (2014) 389-404. 
[25] C. Bianca, Modeling complex systems by functional subsystems representation and thermostatted-KTAP methods, Appl. Math. Inf. Sci 6 (2012) 495-499.

[26] C. Bianca, Modeling complex systems with particles refuge by thermostatted kinetic theory methods, Abstract and Applied Analysis, 2013 (2013) 152174.

[27] K. F. Gauss, Uber ein Neues Allgemeines Grundgesatz der Mechanik (On a New Fundamental Law of Mechanics), J. Reine Angewandte Math. 4 (1829) 232-235.

[28] C. Bianca, M. Ferrara, L. Guerrini, Asymptotic limit of an integro-differential equation modelling complex systems, Izvestiya: Mathematics (2014), to appear.

[29] C. Bianca, C. Dogbe, Kinetic models coupled with gaussian thermostats: Macroscopic frameworks, (2014), submitted.

[30] C. Bianca and M. Pennisi, The triplex vaccine effects in mammary carcinoma: A nonlinear model in tune with simtriplex, Nonlinear Analysis: Real World Applications 13 (2012) 1913-1940.

[31] F. Michor, Y. Iwasa, M.A. Nowak, Dynamics of cancer progression, Nat. Rev. Cancer 4 (2004) 197-205.

[32] C. L. Jorcyk, M. Kolev, K. Tawara, B. Zubik-Kowal, Experimental versus numerical data for breast cancer progression. Nonlinear Analysis RWA 13 (2012) 78-84.

[33] P. Carmeliet, R.K. Jain, Angiogenesis in cancer and other diseases, Nature 407 (2000) 249-257.

[34] J. Folkman and R. Kerbel, Clinical translation to angiogenesis inhibitors, Nature Reviews Cancer 2 (2002) 727-739.

[35] G. P. Morris, C.P. Dettmann, Thermostats: analysis and application, Chaos 8 (1998) 321-336.

[36] D. Ruelle, Smooth dynamics and new theoretical ideas in nonequilibrium statistical mechanics, J. Stat. Phys. 95 (1999) 393-468.

[37] H. G Othmer, T. Hillen, The diffusion limit of transport equations II: Chemotaxis equations, SIAM J Appl Math 62 (2002) 1222-1250.

[38] R. Kapral, Multiparticle collision dynamics: Simulation of complex systems on mesoscales, Adv. Chem. Phys., 140 (2008) 89-146.

[39] N. Bellomo, A. Bellouquid, J. Nieto, J. Soler, On the asymptotic theory from microscopic to macroscopic growing tissue models: An overview with perspectives, Math. Models Methods Appl. Sci. 21 (2012) 1-37.

[40] C. Bianca, How do mutative events modify moments evolution in thermostatted kinetic models?, Communications in Nonlinear Science and Numerical Simulation 19 (2014) 2014. 